\title{
The Validity of Nonlinear Geometric Optics for Weak Solutions of Conservation Laws
}

\author{
Ronald J. DiPerna ${ }^{1}$ and Andrew Majda ${ }^{2}$ \\ Department of Mathematics, Duke University, Durham, North Carolina 27706, USA \\ Department of Mathematics, University of California, Berkeley, California 94720, USA
}

\begin{abstract}
The method of weakly nonlinear geometric optics is one of the main formal perturbation techniques used in analyzing nonlinear wave motion for hyperbolic systems. The tacit assumption in using such perturbation methods is that the corresponding solutions of the hyperbolic system remain smooth; since shock waves typically form in such solutions, these assumptions are rarely satisfied in practice. Nevertheless, in a variety of applied contexts, these methods give qualitatively reliable answers for discontinuous weak solutions. Here we give a rigorous proof for the validity of nonlinear geometric optics for general weak solutions of systems of hyperbolic conservation laws in a single space variable. The methods of proof do not mimic the formal construction of weakly nonlinear asymptotics but instead rely on structural symmetries of the approximating equations, stability estimates for intermediate asymptotic times, and the rapid decay in variation of weak solutions for large asymptotic times.
\end{abstract}

\section{Introduction}

After the early work of Landau [13], Lighthill [9], and Whitham [26], the method of weakly nonlinear geometric optics has evolved into one of the main perturbation techniques for analyzing solutions of quasi-linear hyperbolic equations (see [24] for a review of the applied literature before 1981). There are recent multi-dimensional applications of these methods to the development of simplified models in reacting gas flow [23], to the regular reflection of weak shocks [8], and to the formation of Mach stems in reacting shock fronts [22]. Furthermore, systematic self-consistent derivations using nonlinear geometric optics for simplified theories of both nonresonant and resonant wave interactions in several space dimensions have been developed recently $([9,10,20])$. While the tacit assumptions used in the formal derivation of the expansion of weakly nonlinear geometric optics are that the

1 Partially supported by NSF Grant No. DMS-8301135

2 Partially supported by NSF Grant No. MCS-81-02360 and ARO Grant No. 483964-25530

Current Address: Department of Mathematics, Princeton University, Princeton, N.J. 08544, USA 
solutions of the hyperbolic system are smooth, these methods often yield good approximate solutions even after shocks have developed for general weak solutions of conservation laws. In this paper our main objective is to analyze this somewhat surprising fact and to present rigorous proofs for the validity of the expansions of nonlinear geometric optics for general weak solutions of systems of conservation laws in a single space variable.

We consider solutions $u$ of the $n \times n$ system of hyperbolic conservation laws,

$$
u_{t}+f(u)_{x}=0, \quad u(x, 0)=u_{0}(x) .
$$

We require that the Jacobian matrix, $\nabla f(u)=A(u)$ has $n$ distinct real eigenvalues, $\lambda_{1}(u)<\lambda_{2}(u)<\cdots<\lambda_{n}(u)$ with corresponding right and left eigenvectors $\left\{r_{j}(u)\right\}_{j=1}^{n}$, $\left\{l_{k}(u)\right\}_{k=1}^{n}$ satisfying

$$
A r_{j}=\lambda_{j} r_{j}, \quad l_{k} A=l_{k} \lambda_{k}, \quad l_{k} \cdot r_{j}=\delta_{k j} .
$$

In addition, we require that each wave speed is genuinely nonlinear so that

$$
\nabla_{u} \lambda_{k} \cdot r_{k} \neq 0, \quad k=1, \ldots, n .
$$

The prototypical example of such a system of hyperbolic conservation laws is the $2 \times 2$ system governing isentropic compressible fluid flow,

$$
\rho_{t}+m_{x}=0, \quad m_{t}+\left(\frac{m^{2}}{\rho}+p(\rho)\right)_{x}=0
$$

with $u={ }^{t}(\rho, m), \rho$, the density, $m$, the momentum and $p(\rho)$ determined by an equation of state such as the ideal gas law,

$$
p(\rho)=A \rho^{\gamma}, \quad A>0, \quad \gamma>1 .
$$

Before describing our rigorous results, we include a brief formal derivation of the method of weakly nonlinear geometric optics for systems of conservation laws in a single space variable (see $[10,20]$ for details and generalizations). The constant state $u_{0}$ is a trivial solution of (1.1) and we consider perturbed small amplitude solutions with the form

$$
u(x, t)=u_{0}+\varepsilon \sum_{j=1}^{n} u_{j}\left(\phi^{j}, \tau\right)+\varepsilon^{2} \bar{u}(\vec{\phi}, t, \tau)
$$

where $\tau=\varepsilon t$ is a long time scale and $\phi^{j}(x, t)$ for $1 \leqq j \leqq n$ is a phase function to be determined. We expand the nonlinear flux in (1.1) to second order about $u_{0}$ to get

$$
f\left(u_{0}+\varepsilon v\right)=f\left(u_{0}\right)+\varepsilon A\left(u_{0}\right) v+\frac{\varepsilon^{2}}{2} \nabla_{u}^{2} f\left(u_{0}\right)(v, v)+o\left(\varepsilon^{2}\right) .
$$

With the notation, $u_{j}\left(\theta_{j}, \tau\right)$, we substitute the ansatz from (1.4) into the equation in (1.1) and attempt to construct formal solutions within $o\left(\varepsilon^{2}\right)$. The terms of order $\varepsilon$ vanish provided that

$$
\left(\phi_{t}^{j}+A\left(u_{0}\right) \phi_{x}^{j}\right) \partial_{\theta_{j}} u_{j}=0
$$

for $j=1, \ldots, n$. These equations are satisfied provided that we choose

$$
\phi_{j}=x-\lambda_{j}\left(u_{0}\right) t, \quad u_{j}=\sigma^{j}\left(\theta_{j}, \tau\right) r_{j}\left(u_{0}\right)
$$


for $j=1, \ldots, n$. At this stage in the argument, $\sigma_{j}\left(\theta_{j}, \tau\right)$ is an arbitrary scalar amplitude function. Continuing the expansion, we compute that the ansatz from (1.4) yields a formal solution to order $o\left(\varepsilon^{2}\right)$ provided that $\bar{u}_{2}(\vec{\phi}, \tau)=\sum_{j=1}^{n} \bar{u}_{2}^{j}\left(\theta_{j}, \tau\right)$ with $\bar{u}_{2}^{j}$ satisfying,

$$
\left(\lambda_{j}\left(u_{0}\right) I-A\left(u_{0}\right)\right)\left(\bar{u}_{2}^{j}\right)_{\theta_{j}}=\sigma_{\tau}^{j} r_{j}+\nabla_{u}^{2} f\left(u_{0}\right)\left(r_{j}, r_{j}\right)\left(\frac{1}{2}\left(\sigma^{j}\right)^{2}\right)_{\theta_{j}}
$$

for $j=1, \ldots, n$. The matrix on the left-hand side of (1.6) has zero as a simple eigenvalue with left eigenvector $l_{j}\left(u_{0}\right)$; thus, we can find $\bar{u}_{2}^{j}$ to solve the equations to order $\varepsilon^{2}$ only if the inner product of $l_{j}\left(u_{0}\right)$ and the right-hand side of (1.6) vanishes. This requirement yields a decoupled system of scalar conservation laws for the amplitude functions, $\sigma^{j}$; the $\sigma^{j}(\theta, \tau)$ satisfy

$$
\sigma_{\tau}^{j}+b_{j}\left(\frac{1}{2}\left(\sigma^{j}\right)^{2}\right)_{\theta}=0, \quad 1 \leqq j \leqq n
$$

with the coefficient $b_{j}$ given by $b_{j}=l_{j}\left(u_{0}\right) . \nabla_{u}^{2} f\left(u_{0}\right)\left(r_{j}\left(u_{0}\right), r_{j}\left(u_{0}\right)\right)$. One well known consequence of the genuine-nonlinearity condition from (1.3) is that $b_{j} \neq 0$ for $j=$ $1, \ldots, n$. (See [21].) Thus, the equations in (1.7) are decoupled inviscid Burgers equations. Of course the region of validity of this expansion is further restricted to spacetime domains where $\bar{u}_{2}$ grows sublinearly in $t$. To summarize, we see that the formal expansions of weakly nonlinear geometric optics suggest that smooth solutions of general hyperbolic systems can be approximated within $O\left(\varepsilon^{2}\right)$ by the geometric optics expansion,

$$
u_{w}^{\varepsilon}=u_{0}+\varepsilon \sum_{j=1}^{n} \sigma^{j}\left(\phi^{j}, \tau\right) r_{j}\left(u^{0}\right)
$$

where $\sigma^{j}(\theta, \tau)$ satisfies the much simpler Burgers equation in (1.7) for $j=1, \ldots, n$.

The above derivation obviously relies on the fact that $\sigma_{j}(\theta, \tau)$ is a smooth function (see (1.6) for example) and in contrast, solutions of the inviscid Burgers equation typically develop shocks in finite time. Nevertheless, it was suggested in Chap. 1 of [21] that the expansion in (1.8) should remain valid for times after shock waves have formed. This conjecture was based on the following two facts: the explicit examples analyzed in [21] suggested uniform validity for an arbitrarily large time where the solution remained smooth with an error estimate independent of the first derivatives of the solution; the work of DiPerna [2] and Liu [18] on the detailed large time behavior of solutions of conservation laws gave a rigorous proof that solutions of (1.1) with initial data of compact support approach decoupled $N$-waves as $T \rightarrow \infty$. These $N$-waves are not weak solutions of (1.1) but are instead weak solutions of the decoupled Burgers equations in (1.7).

Here we prove the above conjecture. We consider the general weak solution $u^{\varepsilon}$ for (1.1) (constructed by Glimm's method) with initial data of the form

$$
u^{\varepsilon}(x, 0)=u_{0}+\varepsilon v(x) \text {. }
$$

We consider the corresponding weakly nonlinear asymptotic weak solution from (1.8) where $\sigma^{j}(\theta, \tau)$ is the weak solution of the Burgers equation in (1.7) with initial data,

$$
\sigma^{j}(\theta, 0)=l_{j}\left(u_{0}\right) \cdot v_{0}(x), \quad 1 \leqq j \leqq n
$$


We introduce the $L^{1}$-norm, $|u|_{1}=\int_{R^{1}}|u| d x$ and also denote the $L^{1}$-norm of a periodic function with period $p$ by $|u|_{1}=1 / p \int_{0}^{p}|u| d x$. The remaining sections of this paper contain a proof of the following result:

\section{Theorem. (Justification of Nonlinear Optics)}

A) Assume that $v_{0}(x)$ is an arbitrary function of bounded variation and with compact support. Consider the weak solution $u^{\varepsilon}(x, t)$ for (1.1) with the initial data in (1.9) and the weakly nonlinear geometric optics approximation $u_{w}^{\varepsilon}(x, t)$ defined in $(1.8)$ and (1.10), then we have the estimate,

$$
\max _{0 \leqq t<+\infty}\left|u^{\varepsilon}(\cdot, t)-u_{w}^{\varepsilon}(\cdot, t)\right|_{1} \leqq C \varepsilon^{2},
$$

uniformly for all times where $C$ depends on the support of $v_{0}(x)$ and the derivatives of the flux function, $f(u)$.

B) Assume that $v_{0}(x)$ is a periodic function with bounded variation per period. For any scalar convex conservation law, i.e. $n=1$, we have

$$
\max _{0 \leqq t<+\infty}\left|u^{\varepsilon}(\cdot, t)-u_{w}^{\varepsilon}(\cdot, t)\right|_{1} \leqq C \varepsilon^{3} .
$$

On the other hand, for a pair of conservation laws, i.e. $N=2$, and periodic initial data with bounded variation per period, we have the weaker estimate

$$
\left|u^{\varepsilon}(\cdot, t)-u_{w}^{\varepsilon}(\cdot, t)\right|_{1} \leqq t C \varepsilon^{2} .
$$

In $\mathrm{B})$ the constant $C$ depends on the derivatives of $f$, the estimates for the $L^{\infty}$ norm of $v_{0}(x)$, and the period, $p$. While the estimates for the global uniformly valid approximation of $u_{w}^{\varepsilon}$ to $u^{\varepsilon}$ from (1.11) and (1.12) are much stronger than is anticipated by the formal theory, we remark that the weaker estimate in (1.13) is still sufficient to justify $u_{w}^{\varepsilon}$ as the leading order asymptotic term for periodic waves for $M=2$ and any large time of order $o\left(\varepsilon^{-2}\right)$. These times are much longer than the times of order $O\left(\varepsilon^{-1}\right)$ where smooth $\varepsilon$-amplitude periodic data develop shock waves. On the other hand, the restriction in part (B) of the theorem to $n \leqq 2$ is not merely a technical one; for $n \geqq 3$ and periodic initial data, the decoupled inviscid Burger equations from (1.7) are not sufficient to describe the leading order asymptotics for $u_{w}^{\varepsilon}$ even at the purely formal level due to the appearance of resonant wave interactions (see [20]). An accessible open problem for this more subtle case will be described at the end of this introduction.

Next we give two instructive elementary examples which illustrate several features of the theorem. First we consider small amplitude solutions about the zero background state for scalar convex conservation laws,

$$
u_{t}+f(u)_{x}=0
$$

with $f^{\prime}(u)=a(u), f^{\prime \prime}(u)>0$ and conveniently normalized with $f^{\prime}(0)=0$ and $f^{\prime \prime}(0)=1$. For discontinuous Riemann initial data with the form

$$
u^{\varepsilon}(x, 0)=\left\{\begin{array}{rl}
\varepsilon, & x \leqq 0 \\
-\varepsilon, & x>0
\end{array},\right.
$$


we have the solution $u^{\varepsilon}(x, t)$ given by

$$
u^{\varepsilon}(x, t)=\left\{\begin{aligned}
\varepsilon, & x \leqq s^{\varepsilon} t \\
-\varepsilon, & x \geqq s^{\varepsilon} t
\end{aligned}\right.
$$

with $s^{\varepsilon}=(2 \varepsilon)^{-1}(f(\varepsilon)-f(-\varepsilon))$. Provided that $f^{(3)}(0) \neq 0, s^{\varepsilon}$ satisfies $C^{-1} \varepsilon^{2} \leqq$ $\left|S^{\varepsilon}\right| \leqq C \varepsilon^{2}$ with some $C>0$. For this example, $u_{w}^{\varepsilon}(x, t)$ is given by

$$
u_{w}^{\varepsilon}(x, t)=\left\{\begin{array}{rll}
\varepsilon & x \leqq 0, & t \geqq 0 \\
-\varepsilon & x>0, & t \geqq 0
\end{array} .\right.
$$

Thus, in this particular example, the deviation of $u_{w}^{\varepsilon}$ from $u^{\varepsilon}$ in the maximum norm is always only $O(\varepsilon)$ due to the difference in shock location. On the other hand, if we compute the deviation in the $L^{1}$ norm, we have

$$
C^{-1} t \varepsilon^{3} \leqq\left|u^{\varepsilon}(\cdot, t)-u_{w}^{\varepsilon}(\cdot, t)\right|_{1} \leqq C t \varepsilon^{3} .
$$

For a general system of conservation laws with $n>1$, there will be additional errors due to the production of secondary waves of different wave families. We illustrate this by considering the weakly nonlinear approximation (around $u_{0}=0$ with $\lambda_{k}(0)=0$ for simplicity) for the general system in $(0.1)$ with initial data.

$$
u^{\varepsilon}(x, 0)=\left\{\begin{array}{rr}
\varepsilon r_{k}(0), & x \leqq 0 \\
-\varepsilon r_{k}(0), & x \geqq 0
\end{array}\right\} .
$$

We normalize the right eigenvector by the condition $\nabla \lambda_{k} \cdot r_{k}=1$. We consider any hyperbolic system so that with the above normalization for $r_{k}, l_{j} \cdot\left(\nabla_{u} r_{k}\right) r_{k}(0) \neq 0$ for some $j \neq k$. From this condition, it follows that the centered wave solution $u^{\varepsilon}(x, t)$ of the above Riemann problem necessarily has some waves of strength $O\left(\varepsilon^{2}\right)$ for some $j^{\text {th }}$ wave field with $j \neq k$. The solution $u_{w}^{\varepsilon}(x, t)$ with the above initial data has a similar form to the right-hand side of (1.14) multiplied by $r_{k}(0)$. In this example, the deviation of $u_{w}^{\varepsilon}$ from $u^{\varepsilon}$ has two different sources of error. The first source is an error in amplitude and shock location for the primary wave $j=k$ yielding $L^{1}$ errors of the same form as in (1.15) for the scalar case. The second source of error arises from the fact that $u_{w}^{\varepsilon}$ completely ignores the production of the secondary waves of order $o\left(\varepsilon^{2}\right)$ for $j \neq k$. This second source of error dominates the first, yielding the error estimates for this example,

$$
C^{-1} \varepsilon^{2} t \leqq\left|u^{\varepsilon}(\cdot, t)-u_{w}^{\varepsilon}(\cdot, t)\right|_{1} \leqq C \varepsilon^{2} t
$$

with $C$ nonzero provided that $\nabla \lambda_{k} \cdot r_{k}=1$ and for some $j \neq k, l_{j} \cdot\left(\nabla_{u} r_{k}\right) r_{k}(0) \neq 0$. In this last example, the error behaves in a qualitatively similar fashion as we have stated in part B) of the above theorem for periodic waves and $n=2$. The authors conjecture that the similar weaker estimate in (1.13) for periodic waves is also sharp but the mechanisms are somewhat different than in the above example and are explained via examples in Sect. 6. The stronger estimates in the first parts of the theorem for initial data of compact support and for scalar laws with periodic data occur through the well-known large time cancellation of shock and rarefaction waves of the same family which induces a rapid time decay of the total variation for $u^{\varepsilon}$ and $u^{w}$ (see [7]). The above simple examples also indicate that the resolution (in the sense of [16]) of 
weakly nonlinear approximations is often even better than the integral estimates derived here.

The proof of the theorem does not mimic the classical asymptotic development for smooth solutions described above. Even for scalar convex laws, the proof has three main aspects. First we exploit the fact that solutions of the Burgers equation respect a two parameter symmetry group in addition to uniform space-time dilations and space-time translations; in particular, if $\sigma(x, t)$ is any solution of

$$
\frac{\partial \sigma}{\partial t}+b \frac{\partial}{\partial x}\left(\frac{1}{2} \sigma^{2}\right)=0
$$

then for all constants $u_{0}$ and $\varepsilon>0$,

$$
u=u_{0}+\varepsilon \sigma\left(x-b u_{0} t, \varepsilon t\right)
$$

is also a solution of the Burgers equation. This fact explains the appearance of two distinct time scales in solutions of quadratically nonlinear conservation laws with initial data of the form, $u_{0}+\varepsilon v(x)$ and also trivially justifies weakly nonlinear asymptotics for solutions of the Burgers equation. The second part of the proof for scalar convex laws involves a generalized $L^{1}$-stability estimate for solutions of inhomogeneous scalar conservation laws with inhomogeneous forcing terms given by finite Borel measures. This key lemma is contained in Sect. 3 of the paper and generalizes earlier estimates of this type in $[2,18]$. The $L^{1}$ stability estimate together with the stability of total variation norms is used to control the deviation of $u_{w}^{\varepsilon}$ from $u^{\varepsilon}$ in the beginning and intermediate asymptotic regimes in time. Finally, the third main ingredient in the proof for scalar convex conservation laws uses the rapid decay of total variation for large times and the $L^{1}$ stability estimate to control the deviation of $u^{\varepsilon}$ and $u_{w}^{\varepsilon}$ for large asymptotic times. Section 2 is a preliminary section summarizing facts from the theory of BV functions used throughout the paper while the $L^{1}$ stability lemma is proved in Sect. 3. The proof of the theorem for scalar convex laws and data of compact support is given in Sect. 4 and it uses the tools mentioned above. The proof for systems is given in Sect. 5 in the case $v_{0}(x)$ has compact support and this proof builds on the earlier proof in the scalar case. For systems we exploit the fact developed earlier in $[2,18]$ that weak solutions asymptotically solve decoupled inhomogeneous scalar laws for large times where the inhomogeneous terms are suitably small Borel measurers. Using refined estimates of this decoupling, we reduce to the scalar convex case treated previously. For simplicity in exposition, we only carry out all the details for $n=2$ although it should be clear to the reader that out arguments are readily extended to $n>2$ by incorporating the additional ideas from [19] in a straightforward fashion. Finally, in Sect. 6 we discuss the modifications necessary for treating the periodic case. In contrast to the case when the initial data $v_{0}(x)$ has compact support, it is quite important in Sect. 6 that we have $n=1$ or $n=2$.

We conclude this introduction by pointing out that for periodic initial data, the formal predictions of weakly nonlinear asymptotics do not yield decoupled Burgers equations due to the appearance of resonant wave interactions when $n \geqq 3$. In fact, for the important $3 \times 3$ hyperbolic system describing compressible fluid flow with 
entropy variations, the weakly nonlinear asymptotic equations for the sound waves are two Burgers equations coupled through a linear integral operator with kernel given by the derivative of the initial entropy field (see [20] for the details). One very interesting and accessible open problem is to assess the validity of this approximation for periodic weak solutions of the equations of compressible flow.

\section{The Geometric Structure of Solutions}

In this section we discuss several aspects of the structure theory for discontinuous solutions to hyperbolic conservation laws,

$$
\partial_{t} u+\partial_{x} f(u)=0,
$$

which are relevant to the theory of weakly nonlinear asymptotics. We assume that $f$ is a smooth map from $R^{n}$ to $R^{n}$ whose Jacobian $\nabla f(u)=\left(\partial f^{i} / \partial u_{j}\right)$ has $n$ real and distinct eigenvalues

$$
\lambda_{1}(u)<\lambda_{2}(u) \ldots \lambda_{n}(u)
$$

at each state $u$ in $R^{n}$, and that each eigenvalue is genuinely nonlinear in Lax's sense [14], i.e. the directional derivative in the corresponding right eigendirection never vanishes:

$$
r_{j} \cdot \nabla \lambda_{j} \neq 0, \text { where } \nabla f r_{j}=\lambda_{j} r_{j}
$$

This class of systems includes several classical $2 \times 2$ systems, for example, the equations of isentropic gas dynamics and the equations of shallow water theory.

It is well known that, for systems of this type, the Cauchy problem does not in general possess a globally defined smooth solution even if the initial data are smooth. The nonlinear structure of the eigenvalues leads to the generation of shock waves in a finite time. One must therefore develop an existence theory in the framework weak solutions, i.e. solutions satisfying the conservation laws (2.1) in the sense of distributions:

$$
\iint \partial_{t} \phi u+\partial_{x} \phi f(u) d x d t+\int \phi(x, 0) u(x, 0) d x=0
$$

for all smooth test functions $\phi$ with compact support in the upper half-plane $t \geqq 0$.

The classical prototype of a weak solution is provided by a piecewise smooth solution, i.e. by a solution which is smooth except for a finite number of curves of discontinuity. In mechanics, piecewise smooth solutions have, of course, a natural physical interpretation: the regions of the $x-t$ plane on which the solution is smooth represent rarefaction waves, compression waves and their interactions, while the curves of discontinuity represent propagating shock waves. Although the class of piecewise smooth solutions is fairly wide, it is not sufficiently broad to encompass all solutions, even those with compactly supported $C^{\infty}$ data. As a consequence, one is compelled to entertain wider function spaces for the purpose of constructing and analyzing solutions to hyperbolic conservation laws.

Research in the area of conservation laws in one space dimension has indicated that a natural framework for weak solutions is provided by the space of functions of bounded variation in the sense of Cesari $[5,25]$. We shall recall several features of 
BV functions which are relevant to our analysis of the asymptotic structure of general perturbations.

A real-valued locally integrable function $u=u(y)$ defined on a domain $\Omega \subset R^{m}$ is called a function of bounded variation if each of its first order partial derivatives is represented by a locally finite Borel measure:

$$
\int_{\Omega} u \frac{\partial \phi}{\partial y_{i}} d y=-\int_{\Omega} \phi d \mu_{j}
$$

for all smooth compactly supported functions $\phi$, while the weight which the measure $\mu_{j}$ assigns to an arbitrary compact subset $K \subset \Omega$ is finite. A vector-valued function lies in the space $\mathrm{BV}(\Omega)$ if each of its components satisfies the conditions above.

The relevance of the space BV for systems of conservation laws (2.1) was established by a constructive existence theorem of Glimm [6] for solutions to the Cauchy problem with initial data having small total variation.

Theorem 2.1. If $T V u_{0} \ll 1$, then the random choice method generates a globally defined distributional solution of (2.1) which assumes the initial data $u_{0}$ at $t=0$ and satisfies the following stability estimates:

$$
\begin{aligned}
|u(\cdot, t)|_{\infty} & \leqq \text { const }\left|u_{0}\right|_{\infty}, \\
T V u(\cdot, t) & \leqq \text { const } T V u_{0} .
\end{aligned}
$$

Thus, in one space dimension, the $L^{\infty}$ norm and the total variation norm provide natural metrics in which to measure the amplitude and gradient of the solution, respectively. For each $t$, the amplitude of the solution is bounded by a constant times the amplitude of the data (2.3), while the total amount of wave magnitude in the solution is bounded by a constant times the total amount of wave magnitude in the data (2.4). These sharp-time estimates provide the starting point in the structure theory for solutions to $(2.1)$, $[3,17]$.

With regard to structure we recall that a scalar function $w$ of one variable lies in the space $\mathrm{BV}(R)$ if its classical total variation is finite, after a possible modification on a set of measure zero. The quantity $T V w$ is computed as follows. Consider a finite partition $x_{1}<x_{2}<\cdots<x_{n}$, calculate the sum of the associated increments of $w$,

$$
\sum_{j=1}^{n-1}\left|w\left(x_{j}\right)-w\left(x_{j+1}\right)\right|
$$

and take the supremum over all partitions:

$$
T V w=\sup \left\{\sum_{j=1}^{n-1}\left|w\left(x_{j}\right)-w\left(x_{j+1}\right)\right|:\left\{x_{j}\right\} \in R\right\} .
$$

From the classical viewpoint, the simplest example of a BV function of one variable is provided by a piecewise constant function with a finite number of jumps. The distributional derivative is represented by a measure with point masses located at the jump points; the total variation is given by the sum of the heights of the jumps. On the other hand, if $w$ is smooth, the distributional derivative is represented by a measure which is absolutely continuous with respect to Lebesgue measure, namely $w^{\prime}(x) d x$, and the total variation reduces to the $L^{1}$ norm of the first derivative: 
$T V w=\int_{-\infty}^{\infty}\left|w^{\prime}(x)\right| d x$. A mixture of these two cases useful for many engineering purposes is presented by the piecewise smooth category. Here the total variation norm registers the sum of the jumps plus the $L^{1}$ norm of the first derivative restricted to the intervals of smoothness:

$$
T V w=\sum\left|[w]_{j}\right|+\sum \int_{x_{j}}^{x_{j+1}}\left|w^{\prime}(x)\right| d x,
$$

where

$$
[w]_{j} \equiv \lim _{x \uparrow x_{j}} w-\lim _{x \downarrow x_{j}} w
$$

and $x_{j}$ specify the jump points. In the setting of a piecewise smooth solution to a system of conservation laws (2.1) restricted to a fixed time $t$, i.e. $u=u(\cdot, t)$, the first term in (2.5) represents the sum of the shock strengths in $u$ at time $t$, while the second term measures the fluctuations in the rarefaction waves and compression waves at time $t$. In general, the profile of the solution $u$ at a fixed time $t$ presents a function of bounded variation in $x$ with a more complex structure.

In the geometric theory of conservation laws, one of the central problems concerns the structure and regularity of the solution as a function of $x$ and $t$ jointly. In this regard, the calculus of BV functions proves to be quite useful. Although the space BV is rather broad, there exists a strong analogy between the measuretheoretic structure of general BV functions and the classical pointwise structure of special piecewise smooth functions. Indeed, the subject of geometric measure theory has established a variety of qualitative and quantitative links between the BV category and the piecewise smooth category. We shall conclude this section with a brief discussion of several aspects of the BV structure theory and the BV calculus which are relevant to the computations associated with a rigorous treatment of weakly nonlinear asymptotics in the setting of discontinuous solutions.

Suppose $w$ is a function in $\operatorname{BV}(\Omega), \Omega \subset R^{m}$. It turns out that, with the possible exception of a set with zero $(m-1)$-dimensional Hausdorff measure, each point in the domain of definition $\Omega$ is either a point of approximate continuity or a point of approximate jump discontinuity. At a point $p$ of approximate continuity the average behavior is controlled and locally isotropic: the function $w$ admits an approximate limit with respect to every half-plane through $p$ and all such approximate limits are equal. In contrast, at a jump point $p$, the function $w$ experiences a first-order discontinuity in a unique direction $v ; w$ has distinct approximate limits $L_{v} w$ and $L_{-v} w$ with respect to complementary left and right half-planes through $p$ determined by $v$.

The technical formulation of these properties runs as follows.

At a point of approximate continuity, the $m$-dimensional measure of the set of points where $w$ deviates from $w(p)$ by some fixed amount, inside a ball $B_{r}(p)$ or radius $r$ centered at $p$, vanishes faster than the $m$-dimensional measure of the ball as the radius shrinks:

$$
\lim _{r \rightarrow 0} r^{-m} \int_{B_{r}(p)}|w(x)-w(p)| d x=0 .
$$

At a jump point, there exist two distinct values $w^{ \pm}(p)$ and a unique direction $v$ such 
that

where

$$
\lim _{\boldsymbol{r} \rightarrow 0} r^{-m} \int_{\boldsymbol{B}_{r} \cap \boldsymbol{H}_{v}^{ \pm}}\left|w(x)-w^{ \pm}(p)\right| d x=0,
$$

$$
H_{v}^{ \pm}\{x:(x-p, v) \geqq 0\} \quad \text { and } \quad w^{ \pm}(p)=L_{ \pm v} w(p) .
$$

The overall picture of the geometric structure of a BV function is the following. Most of the domain of definition is filled with points of approximate continuity and firstorder jump discontinuity. In addition, the jump points are organized inside sets with a differentiable structure: the set $\Gamma$ of points of approximate jump discontinuity forms an at most countable union of rectifiable sets of dimension $m-1$, i.e.

$$
\Gamma=\bigcap_{j=1}^{\infty} \Gamma_{j}
$$

where $\Gamma_{j}$ is the image of some compact subset of $R^{m-1}$ under some Lipschitz map from $R^{m-1}$ to $R^{m}$. By analogy with fluid dynamics it is standard to refer to $\Gamma$ as the shock set of $w$ : the set $\Gamma^{\mathrm{c}}$ is reminiscent of the "continuous" region of flow, the set recalls the propagating discontinuities.

Finally we shall recall several theorems in the BV calculus. For the purposes of this paper it suffices to work with functions in $\mathrm{BV} \cap L^{\infty}$.

Theorem 2.2. If $w \in \mathrm{BV} \cap L^{\infty}\left(R^{m}\right)$ and $E$ is a bounded subset with finite perimeter, then the weight which the gradient of $w$ assigns to $E$ is expressible as a boundary integral with respect to $(m-1)$-dimensional Hausdorff measure,

$$
\frac{\partial w}{\partial x_{j}}(E)=\int_{\partial^{*} E} w v_{j} d H_{m-1},
$$

where $v=\left(v_{1}, \ldots, v_{n}\right)$ represents the outward unit normal to the essential boundary $\partial^{*} E$ of $E$ and $w^{-}$is the inward trace of $w^{-}$on $\partial^{*} E$.

If $w$ is smooth, then formula (2.6) assumes the classical form of Green's Theorem:

$$
\int_{E} \nabla w(x) d x=\int_{\partial^{*} E} w^{-} v d H_{m-1} .
$$

In general, the inward trace $w^{-}$coincides with $w$ at points of approximate continuity and with the half-plane limit $L_{-v} w$ at points of approximate jump discontinuity, with the possible exception of a subset of $\partial^{*} E$ having zero $(m-1)$-dimensional Hausdorff measure.

The second main form of Green's Theorem describes the weight which the gradient assigns to sets with codimension one.

Theorem 2.3. If $w \in \mathrm{BV} \cap L^{\infty}\left(R^{n}\right)$ and $S$ is a Borel set which can be covered by a countable union of essential boundaries of sets with finite perimeter, then

$$
\nabla w(S)=\int_{S}[w]_{v} d H_{m-1},
$$

where

$$
[w]=L_{v} w-L_{-v} w
$$


We observe that the jump quantity $v[w]$ is well-defined, with the exception of an insignificant set with zero $(m-1)$-dimensional Hausdorff measure.

In conclusion we record the generalized chain rule for $\mathrm{BV}$ functions which plays a major role in the derivation of the generalized characteristic equations for $\mathbf{B V}$ solutions.

Theorem 2.4. If $w \in \mathrm{BV} \cap L^{\infty}\left(R^{n}\right)$ and $f: R \rightarrow R$ is smooth then $f(w) \in \mathrm{BV} \cap L^{\infty}$ and

$$
\frac{\partial}{\partial x_{i}} f(w)=\hat{f}^{\prime}(u) \frac{\partial}{\partial x_{i}} w
$$

where the mean value of the derivative of $f$ is defined by the equation

$$
\hat{f}^{\prime}=\int_{0}^{1} f^{\prime}\left\{s L_{v} w(x)+(1-s) L_{-v} w(x)\right\} d s,
$$

and where the equality in (1.8) is understood in the sense of Borel measures.

\section{The Stability of Solutions to Scalar Conservation Laws}

In this section we shall present a general stability theorem for BV solutions to inhomogeneous scalar conservation laws. For simplicity in notation we shall treat the problem in one space dimension; the extension to several dimensions is immediate.

Suppose $f: R \rightarrow R$ is an arbitrary smooth map and $u_{1}, u_{2}$ is an arbitrary pair of functions in $\mathrm{BV} \cap L^{\infty}\left(R^{2}\right)$. Let $\mu_{j}$ denote the Borel measure corresponding to the divergence of the field $\left(u_{j}, f\left(u_{j}\right)\right)$ :

$$
\mu_{j} \equiv \partial_{t} u_{j}+\partial_{x} f\left(u_{j}\right)
$$

Given two times $t_{1}<t_{2}$, the problem is to estimate $L^{1}$ difference between $u_{1}$ and $u_{2}$ at time $t_{2}$ in terms of three quantities: the $L^{1}$ difference at time $t_{1}$, the total mass of $\mu_{j}$ in the closed strip $\left[t_{1}, t_{2}\right]$ and the magnitude of an appropriate entropy field. In order to view the problem in a general context, we shall briefly discuss the notion of generalized entropy for systems of conservation laws in one space dimension,

$$
\partial_{t} u+\partial_{x} f(u)=0, \quad u=u(x, t) \in R^{n},
$$

and then adapt the discussion to the scalar setting.

Definition. A pair $(\eta, q)$ of real-valued maps on the state space $R^{n}$ is called an entropy pair of system (3.2) if all smooth solutions $u(x, t)$ satisfy an additional conservation law of the form

$$
\partial_{t} \eta(u)+\partial_{x} q(u)=0
$$

Thus, a pair of state variables serves as an entropy pair if the divergence of the corresponding entropy field $\{\eta(u(x, t)), q(u(x, t))\}$ vanishes for all smooth flows $u(x, t)$.

The existence of an entropy pair is based upon the solvability of a differential compatibility condition in the state space $R^{n}$, which takes the form

$$
\nabla \eta \nabla f=\nabla q, \quad \text { or equivalently, } \quad \sum_{i} \frac{\partial \eta}{\partial u_{i}} \frac{\partial f^{i}}{\partial u_{j}}=\frac{\partial q}{\partial u_{j}},
$$


and which is derived as follows. Suppose $u(x, t)$ is a smooth solution to (3.2). Writing (3.2) and the entropy identity (3.3) in quasilinear form,

$$
\partial_{t} u+\nabla f(u) \partial_{x} u=0 ; \quad \nabla \eta(u) \partial_{t} u+\nabla q(u) \partial_{x} u=0,
$$

leads to the formal equation

$$
(-\nabla \eta \nabla f+\nabla q) u_{x}=0,
$$

which holds for all smooth solutions $u$ if and only if the coefficient vanishes identically. One therefore deduces a compatibility condition between the generalized entropy $\eta$ and the generalized entropy flux $q$, in the form (3.4) of a first order linear system of $n$ differential equations for two unknowns. In the setting of a scalar equation $(n=1)$ the relation is underdetermined; $\eta$ may be chosen arbitrarily provided that the companion flux $q$ is defined by

$$
q(u)=\int^{u} \eta^{\prime}(u) f^{\prime}(u) d u .
$$

In the setting of systems of two equations, the compatibility condition represents a determined first order linear hyperbolic system [15] and therefore has many solutions. In the setting of mechanics, the existence of an entropy pair is both familiar and explicit: for example any smooth flow $\{\rho(x, t), m(x, t)\}$ conserving mass and momentum in the form of system (1.3) also conserves mechanical energy, i.e.

$$
\partial_{t} E+\partial_{x}(u E+u p)_{x}=0 ; \quad E=\frac{1}{2} \rho u^{2}+\rho \varepsilon(\rho),
$$

where $\varepsilon$ denotes the specific internal energy at a fixed value of the thermodynamic entropy. Thus, for insentropic gas dynamics, the total energy $E$ plays the role of a generalized entropy $\eta$, while the transport term $u E$ plus the work done by the pressure field plays the role of a generalized entropy flux $q$. For systems of more than two equations, the existence of an entropy pair is a rare event which fortunately occurs for the basic systems of continuum mechanics. Furthermore, in the setting of mechanics, the "natural" generalized entropy $\eta$ is a strictly convex function of $u$, in terms of which the physically meaningful solutions are distinguished from the class of all possible weak solutions through the following admissibility criterion.

Definition. A weak solution $u=u(x, t)$ of a system of conservation laws (3.1), endowed with an entropy pair $(\eta, q)$ for which $\eta$ is strictly convex, is called admissible if the divergence of the corresponding entropy field is nonpositive:

$$
\partial_{t} \eta(u)+\partial_{x} q(u) \leqq 0 .
$$

In the case of isentropic gas dynamics, strict hyperbolicity of the system (1.3) corresponds to the condition that $p^{\prime}(\rho)>0$, and thus to the presence of a strictly convex generalized entropy

$$
\eta \equiv E=\frac{1}{2} \rho u^{2}+\rho \varepsilon(\rho)=\frac{1}{2} m^{2} / \rho+\rho \varepsilon(\rho), \quad d \varepsilon=p / \rho^{2} d \rho,
$$

in terms of which admissibility of the solution reduces to the statement that all of its shock waves dissipate mechanical energy.

Remark. It can be shown that the notion of admissibility is well-defined, at least 
for solutions with small oscillation: if (3.5) holds for one entropy pair $(\eta, q)$ with $\nabla^{2} \eta>0$, then it holds for all entropy pairs $(\eta, q)$ with $\nabla^{2} \eta \geqq 0$.

In the setting of a genuinely nonlinear system, it can be shown that a single entropy inequality of the form (3.5) is sufficiently powerful to rule out all nonphysical solutions. However, if the system is not genuinely nonlinear, then additional constraints are required. At the present time it is only in the setting of a scalar equation that a complete understanding of the proper formulation of an entropy admissibility criterion is available together with its role in inducing stability of solutions.

Definitions. A weak solution $u$ to a general (non-convex) scalar conservation law is called admissible if

$$
\partial_{t} \eta(u)+\partial_{x} q(u) \leqq 0
$$

for all convex functions $\eta$, where the corresponding flux is given by (3.5).

Theorem. If $u_{1}$ and $u_{2}$ are two admissible solutions to a general scalar conservation law in $\mathrm{BV} \cap L^{\infty}\left(R^{2}\right)$, then

$$
\int_{-\infty}^{\infty}\left|u_{1}(x, t)-u_{2}(x, t)\right| d x \leqq \int_{-\infty}^{\infty}\left|u_{1}(x, 0)-u_{2}(x, 0)\right| d x .
$$

This $L^{1}$ stability theorem was established by Vol'pert [25] and subsequently generalized by Kruzkov [12] to the $L^{\infty}$ category. An independent proof was given by Keyfitz [11] in the category of piecewise smooth solutions. Our purpose here is to extend the analysis to inhomogeneous scalar equations

$$
\partial_{t} u+\partial_{x} f(u)=\mu,
$$

where $\mu$ is a locally finite Borel measure.

Several preliminary remarks are in order concerning the structure of the entropy fields for a general solution $u$ in $\mathrm{BV} \cap L^{\infty}$. We recall that if $(\eta, q)$ is an arbitrary entropy pair for system (3.1) and if $u$ is an arbitrary solution of (3.1) in BV $\cap L^{\infty}$, then the associated dissipation measure $\beta_{u} \equiv \partial_{t} \eta(u)+\partial_{x} q(u)$ is concentrated on the shock set $\Gamma(u)$ of $u$, i.e. $\beta_{u}(E)=\beta_{u}(E \cap \Gamma)$ for all Borel sets $E$, and may be represented by integration, $\beta_{u}(S)=\int_{S} v_{t}[\eta]+v_{x}[q] d H_{1}$, when restricted to Borel subsets $S \subset \Gamma$. Here the local rate of entropy change is given by

$$
v_{t}[\eta]+v_{x}[q] \equiv v_{t}\left\{L_{v} \eta-L_{-v} \eta\right\}+v_{x}\left\{L_{v} q-L_{-v} q\right\}
$$

at points of jump discontinuity. Both facts are straightforward consequences of the generalized version of Green's Theorem for BV functions and the generalized chain rule, cf. Sect. 2. We refer the reader to [4] for details of the derivation. One may therefore formulate a local version of the admissiblity criterion for BV solutions to a general scalar laws as follows:

Definition. A solution $u \in \mathrm{BV} \cap L^{\infty}$ of a general scalar law is admissible if for all convex functions $\eta$ the inequality

$$
v_{t}[\eta]+v_{x}[q] \leqq 0
$$

holds at $H_{1}$-almost all points of the shock set $\Gamma(u)$. 
Remark 1. In the setting of a scalar law there is a variety of ways to formulate the entropy condition. One could take, for example, any (countable) set of convex functions whose closed convex hull generates the space of all convex functions and postulate the entropy inequality (3.6), (3.9) just for those. The general case would follow by closure. Indeed this is frequently done, with a simple standard choice provided by the collection of all absolute value functions $\eta(u, k)=|u-k|$, together with the affine maps $\eta=a u+b$. Thus, a solution $u$ of a scalar law is admissible if

$$
\partial_{t}(|u-k|)+\partial_{x}(\operatorname{sgn}(u-k)\{f(u)-f(k)\}) \leqq 0
$$

for all $k$, or equivalently for all rational $k$.

Remark 2. The entropy pair

$$
\eta(u, k)=|u-k|, \quad q(u, k)=\operatorname{sgn}(u-k)\{f(u)-f(k)\}
$$

plays a distinguished role in the subject of stability for scalar laws due to the symmetric dependence of $\eta$ and $q$ on $u$ and $k$ : for each $k,(\eta, q)$ is an entropy pair in $u$ and for each $u,(\eta, q)$ is an entropy pair in $k$. The presence of symmetric entropy pair is a distinguishing feature of the scalar equation and is the ultimate source for the $L^{1}$ contraction property (3.7) of the solution operator. This symmetry is an essential ingredient in analyzing stability for the inhomogeneous equation.

Remark 3. From the piecewise linear entropies, one can easily derive Oleinik's original form of the entropy condition which requires that a jump discontinuity propagating at speed $s$ and joining two states $u^{-}$and $u^{+}$from left to right have the following property. The graph of $f$ lies below the chord joining $\left(u^{-}, f\left(u^{-}\right)\right)$to $\left(u^{+}, f\left(u^{+}\right)\right)$whenever $u^{-}>u^{+}$, and above the chord whenever $u^{-}<u^{+}$, i.e.

$$
\frac{f\left(u^{+}\right)-f(k)}{u^{+}-k} \leqq s \leqq \frac{f\left(u^{-}\right)-f(k)}{u^{-}-k}
$$

for all $k$ in the interval between $u^{-}$and $u^{+}$, where $s$ is given by the RankineHugoniot relations,

$$
s=\frac{f\left(u^{+}\right)-f\left(u^{-}\right)}{u^{+}-u^{-}} .
$$

In the case of a BV solution, the speed of propagation associated with a jump point with normal $v$ is defined as the negative ratio of components: $s=-v_{t} / v_{x}$.

Remark 4. For general flux functions $f$, admissibility implies the standard Lax geometric entropy inequality

$$
\begin{gathered}
\lambda\left(u^{+}\right)<s<\lambda\left(u^{-}\right), \quad \lambda \equiv f^{\prime}(u), \\
u^{+}=\lim _{\varepsilon \downarrow 0} u(x+\varepsilon, t), \quad u^{-}=\lim _{\varepsilon \uparrow 0} u(x-\varepsilon, t),
\end{gathered}
$$

asserting that nearby characteristics run into the shock in the forward direction of time. For convex $f,(3.10)$ reduces to the statement that the left-hand limit $u^{-}$exceed the right-hand limit $u^{-}$.

We shall now turn to the stability problem for BV solutions to an inhomogeneous scalar law (3.8). For the purpose of estimating the $L^{1}$ deviation between 
solutions it is natural to consider the maximal rate of entropy production associated with the canonical pair $\left(\eta_{k}, q_{k}\right)$. For each triple $\left(v, u^{-}, u^{+}\right)$, let

$$
\tau(v, u, k)=v_{t}\left[\eta_{k}\right]+v_{k}\left[q_{k}\right] \text { and } \tau(v, u)=\sup _{k} \tau(v, u, k) .
$$

We observe that if $k$ does not lie in the interval $I\left(u^{-}, u^{+}\right)$with ends points $u^{-}$and $u^{+}$, then the rate $\tau(v, u, k)$ of entropy production in $\left(\eta_{k}, q_{k}\right)$ is independent of $k$ and reduces to the Rankine-Hugoniot form:

$$
\begin{aligned}
\tau(v, u, k) & =v_{t}\left\{\eta_{j}\left(u^{+}\right)-\eta_{k}\left(u^{-}\right)\right\}+v_{x}\left\{q_{k}\left(u^{+}\right)-q_{k}\left(u^{-}\right)\right\} \\
& =R(v, u) \equiv v_{t}\left(u^{+}-u^{-}\right)+v_{x}\left\{f\left(u^{+}\right)-f\left(u^{-}\right)\right\}
\end{aligned}
$$

Thus, the supremum is well-defined and we may introduce a maximal measure $\theta$ concentrated on the shock set,

$$
\theta(E)=\int_{E \cap \Gamma(u)} \tau(v, u) d H_{1},
$$

in terms of which we shall establish the following stability theorem.

Theorem 3.1. If $u_{1}$ and $u_{2}$ are arbitrary functions in $\mathrm{BV} \cap L^{\infty}\left(R^{2}\right)$, then

$$
\int_{-\infty}^{\infty}\left|u_{1}(x, t)-u_{2}(x, t)\right| d x \leqq \int_{-\infty}^{\infty}\left|u_{1}(x, \tau)-u_{2}(x, \tau)\right| d x+\sum \theta_{j}(\mathscr{S})+\sum\left|\mu_{j}\right|(\mathscr{S}),
$$

where $\theta_{j}$ is the maximal measure associated with $u_{j},\left|\mu_{j}\right|$ denotes the total variation measure of the divergence field (3.1), and $\mathscr{S}$ denotes the closed strip $[\tau, t]$.

For a genuinely nonlinear equation, the contribution from $\theta_{j}$ may be dominated by $\mu_{j}$.

Corollary 3.1. If $f^{\prime \prime}>0$ and if $u_{j}$ is admissible in the sense that $u_{j}^{-}>u_{j}^{+}$, at $H_{1^{-}}$ almost all points of $\Gamma\left(u_{j}\right)$, then

$$
\int_{-\infty}^{\infty}\left|u_{1}-u_{2}\right|(t) d x \leqq \int_{-\infty}^{\infty}\left|u_{1}-u_{2}\right|(\tau) d x+2 \sum\left|\mu_{j}\right|(\mathscr{S})
$$

Proof of Corollary. For an arbitrary triple $\left(v, u^{-}, u^{+}\right)$, let $s$ and $\sigma$ denote the associated speed of propagation and slope of chord respectively,

$$
s=-v_{t} / v_{x}, \quad \sigma=\frac{f\left(u^{+}\right)-f\left(u^{-}\right)}{u^{+}-u^{-}} .
$$

We note that $s$ and $\sigma$ coincide if and only if the Rankine-Hugoniot form vanishes, i.e.

$$
R(v, u) \equiv v_{t}[u]+v_{x}[f]=0 .
$$

As a consequence of the definitions, we have

$$
s=\sigma-R / v_{x}[u],
$$

and the following identity for the rate of entropy production associated with an arbitrary pair $(\eta, q)$ :

$$
\begin{aligned}
v_{t}[\eta]+v_{x}[q] & =-v_{x}\{s[\eta]-[q]\} \\
& =-v_{x}\{\sigma[\eta]-[q]\}+R(v, u)[\eta] /[u] .
\end{aligned}
$$


In the special case of a piecewise linear entropy $\eta_{k}$, a simple upper bound holds,

$$
v_{t}\left[\eta_{k}\right]+v_{x}\left[q_{k}\right] \leqq-v_{x}\left\{\sigma\left[\eta_{k}\right]-\left[q_{k}\right]\right\}+|R(v, u)| .
$$

If $f^{\prime \prime}>0$, the standard entropy inequality $[u] \geqq 0$ implies that

$$
-v_{x}\left\{\sigma\left[\eta_{k}\right]-\left[q_{k}\right]\right\} \leqq 0, \text { for } k \in I\left(u^{-}, u^{+}\right),
$$

and we conclude that $\tau(v, a) \leqq|R(v, u)|$. Thus, if $E$ is an arbitrary measurable subset of $\Gamma\left(u_{j}\right)$, then

$$
\theta_{j}(E)=\int_{E} \tau\left(v, u_{j}\right) d H_{1} \leqq \int_{E}\left|\tau\left(v, u_{j}\right)\right| d H_{1} \leqq \int_{E}\left|R\left(v, u_{j}\right)\right| d H_{1} .
$$

The corollary follows from the fact that

$$
\int_{E}\left|R\left(v, u_{j}\right)\right| d H_{1}=\left|\mu_{j}\right|(E) .
$$

One may deduce (3.13) from the form of Green's theorem given by (2.7): the weight which the divergence structure measure

$$
\mu_{j}=\partial_{t} u_{j}+\partial_{x} f\left(u_{j}\right) .
$$

Assigns to a subset $E$ of the shock set $\Gamma\left(u_{j}\right)$ admits the representation

$$
\mu_{j}(E)=\int_{E} v_{t}\left[u_{j}\right]+v_{x}\left[f\left(u_{j}\right)\right] d H_{1} \equiv \int_{E} R\left(v, u_{j}\right) d H_{1} .
$$

The total variation measure for any (signed) measure represented by integration is given by the integral of the absolute value of the kernel.

Proof of Theorem 3.1. Following Vol'pert [25], we introduce the divergence $D$ of the vector field

$$
\sigma\left(u_{1}-u_{2}, f\left(u_{1}\right)-f\left(u_{2}\right)\right),
$$

where $\sigma=\operatorname{sgn}\left(u_{1}-u_{2}\right)$ and obtain from Green's Theorem the identity

$$
\begin{aligned}
& \int_{-\infty}^{\infty}\left|u_{1}(t)-u_{2}(t)\right| d x-\int_{-\infty}^{\infty}\left|u_{1}(\tau)-u_{2}(\tau)\right| d x=D(\mathscr{S}), \\
& D=\partial_{t}\left(\left|u_{1}-u_{2}\right|\right)+\partial_{x}\left(\operatorname{sgn}\left(u_{1}-u_{2}\right)\left\{f\left(u_{1}\right)-f\left(u_{2}\right)\right\}\right),
\end{aligned}
$$

where $\mathscr{S}$ denotes the strip $[\tau, t]$. The measure $D$ may be decomposed into two parts related to the forcing measures $\mu_{j}$ and the entropy condition with the aid of the following theorem of Vol'pert [25]. Suppose $u$ and $v$ lie in BV $\cap L^{\infty}\left(R^{m}\right)$ and satisfy $|v| \leqq M|u|$ for some constant $M$. If $s=\operatorname{sgn} u(x)$ then $s u$ is a BV function for which the measures

$$
\frac{\partial}{\partial x_{j}}(s u) \text { and } \bar{s} \frac{\partial}{\partial x_{j}} u
$$

coincide on the set $\Gamma^{c}(u)$. Here $\bar{s}$ denotes the symmetric mean of $s$ obtained by smoothing with a standard symmetric mollifying kernel $\psi_{\varepsilon}$ :

$$
\bar{s}=\lim _{\varepsilon \rightarrow 0} s * \psi_{\varepsilon} .
$$


Thus, the derivatives of the function $\operatorname{sgn} u(x)$ are effectively zero on the set where $u$ is approximately continuous, a fact which facilitates the analysis of the measure $D$ in the following way. With the choices

and

$$
u=u_{1}-u_{2}, \quad v=u_{1}-u_{2}
$$

$$
u=u_{1}-u_{2}, \quad v=f\left(u_{1}\right)-f\left(u_{2}\right),
$$

we may split $D$ as follows

$$
D=D\left|\Gamma\left(u_{1}-u_{2}\right)+D\right| \Gamma^{c}\left(u_{1}-u_{2}\right),
$$

where

$$
D \mid \Gamma^{c}\left(u_{1}-u_{2}\right)=\bar{\sigma}\left\{\partial_{t}\left(u_{1}-u_{2}\right)+\partial_{x}\left(f\left(u_{1}\right)-f\left(u_{2}\right)\right)\right\}=\bar{\sigma}\left(\mu_{1}-\mu_{2}\right),
$$

and the vertical line denotes restriction to the indicated set. Since $|\bar{\sigma}| \leqq 1, D$ may be estimated from above in terms of the total variation measures of $\mu_{j}$,

$$
D \leqq D\left|\Gamma\left(u_{1}-u_{2}\right)+\right| \mu_{1}|+| \mu_{2} \mid,
$$

reducing the problem to one of showing that the restriction of $D$ to the jump set of $u_{1}-u_{2}$ is dominated by the maximal measures, i.e.

$$
D(E) \leqq \theta_{1}(E)+\theta_{2}(E)
$$

for all $E \subset \Gamma\left(u_{1}-u_{2}\right)$.

From the second form of Green's Theorem it follows that

$$
\begin{aligned}
D(E) & =\int_{E} v_{t}\left[\left|u_{1}-u_{2}\right|\right]+v_{x}\left[Q\left(u_{1}, u_{2}\right)\right] d H_{1}, \\
Q\left(u_{1}, u_{2}\right) & \equiv \operatorname{sgn}\left(u_{1}-u_{2}\right)\left\{f\left(u_{1}\right)-f\left(u_{2}\right)\right\},
\end{aligned}
$$

for $E \subset \Gamma\left(u_{1}-u_{2}\right)$. As a consequence of the symmetry of the integrand $I$, we may split $I$ into two terms such that one of the two functions is held fixed in each:

$$
I=v_{t}\left[\left|u_{1}-u_{2}^{+}\right|\right]+v_{x}\left[Q\left(u_{1}, u_{2}^{+}\right)\right]+v_{t}\left[\left|u_{2}-u_{1}^{-}\right|\right]+v_{x}\left[Q\left(u_{2}, u_{1}^{-}\right)\right] .
$$

Thus by appealing to two special choices of $k$, namely $k=u_{2}^{+}$and $k=u_{1}^{-}$, we obtain the desired upper bound $I \leqq \tau\left(v, u_{1}\right)+\tau\left(v, u_{2}\right)$.

\section{Weakly Nonlinear Asymptotics for Scalar Laws}

We are concerned with the response of the solution operator of a scalar genuinely nonlinear equation

$$
\partial_{t} u+\partial_{x} f(u)=0, \quad f^{\prime \prime}>0
$$

to compactly supported small amplitude perturbations of constant initial data:

$$
u^{\varepsilon}(x, 0)=u_{0}+\varepsilon u_{1}(x) .
$$

The classical theory treats $C^{1}$ perturbations $u_{1}(x)$ and postulates a response in the form of a regular multiple scale expansion

$$
u^{\varepsilon}(x, t)=u_{0}+\varepsilon u_{1}(x, t, \tau)+\varepsilon^{2} u_{2}(x, t, \tau)+\ldots,
$$


involving a slow variable $\tau=\varepsilon t$, for an interval of time of order $1 / \varepsilon$ prior to shock development.

Several remarks are in order concerning the impact of symmetry groups on the structure of the expansion. For simplicity, we shall begin by contrasting the general law (4.1) with the special Burgers equation

$$
\partial_{t} u+\partial_{x}\left(b u^{2}\right)=0
$$

based on a quadratic nonlinearity. For the general law (4.1) the symmetry group consists of translations in space and time together with uniform dilations in space-time: if $u(x, t)$ is a solution then so are $u(x+\delta, t), u(x, t+\delta)$, and $u(\delta x, \delta t)$. For the special law (4.2), two additional one-parameter families are present in the form

$$
u(x, t, c)=c+u(x-b c t, t)
$$

reflecting Galilean invariance and

$$
u(x, t, \varepsilon)=\varepsilon^{\alpha} u\left(\varepsilon^{\beta} x, \varepsilon^{\alpha+\beta} t\right),
$$

reflecting joint scaling invariance. Thus, for a second order flux

$$
f(u)=b u^{2},
$$

the solution operator commutes with a two-parameter group of linear transformations acting on the product of physical space and state space. The special symmetry

$$
u(x, t, \varepsilon)=\varepsilon u(x, \varepsilon t)
$$

can be seen to induce the two-scale structure of the leading term of the asymptotic expansion.

A second consequence of the invariance (4.4) is that the formal ansatz of weakly nonlinear asymptotics becomes an exact solution in the case of a one-term expansion applied to Burgers equation, a fact which can be demonstrated as follows. The standard expansion for a general law takes the form

$$
u^{\varepsilon}(x, t)=u_{0}+\varepsilon \sigma\left(x-\lambda_{0} t, \varepsilon t\right)+O\left(\varepsilon^{2}\right)
$$

where, by definition, the perturbation $\sigma$ satisfies the quadratic law

$$
\partial_{t} \sigma+\partial_{x}\left(b \sigma^{2}\right)=0, \quad b=f^{\prime \prime}\left(u_{0}\right) / 2
$$

with rescaled initial data $\sigma(x, 0)=u_{1}(x)$ and $\lambda_{0}=f^{\prime}\left(u_{0}\right)$. For a second order flux (4.3), the group invariance (4.4) implies that the exact solution to the Cauchy problem with perturbed data

$$
u(x, 0)=u_{0}+\varepsilon u_{1}(x)
$$

is given by

$$
u^{\varepsilon}(x, t)=u_{0}+\varepsilon \sigma\left(x-\lambda_{0} t, \varepsilon t\right),
$$

where $\sigma$ solves the canonical law (4.5) with $b=f^{\prime \prime}\left(u_{0}\right) / 2$ and initial data $\sigma(x, 0)=$ 
$u_{1}(x)$. Thus the first two terms of the weakly nonlinear expansion, namely

$$
u_{w}^{\varepsilon}(x, t) \equiv u_{0}+\varepsilon \sigma\left(x-\lambda_{0} t, \varepsilon t\right)
$$

is an exact solution for the problem (4.3), (4.6).

With this fact in mind, one may regard a small amplitude solution of the general law (4.1) as a solution of an inhomogeneous second order law

$$
\partial_{t} u+\partial_{x} Q f\left(u, u_{0}\right)=-\partial_{x} c
$$

where $Q f$ denotes the quadratic part of $f$ at $u_{0}$ and $c$ the cubic residual:

$$
\begin{aligned}
Q f\left(u, u_{0}\right) & \equiv f\left(u_{0}\right)+f^{\prime}\left(u_{0}\right)\left(u-u_{0}\right)+\frac{f^{\prime \prime}\left(u_{0}\right)}{2}\left(u-u_{0}\right)^{2}, \\
c & \equiv f-Q f=O\left(u-u_{0}\right)^{3} .
\end{aligned}
$$

The problem is therefore to compare a solution of the inhomogeneous law (4.8) with the solution of the corresponding homogeneous equation $\partial_{t} u+\partial_{x} Q f=0$ with the same initial data, $u_{0}+\varepsilon u_{1}(x)$, using the uniform $L^{1}$ stability theorem of Sect. 3.

The main result of this section is the following uniform approximation theorem for compactly supported perturbations.

Theorem 4.1. Suppose $u_{1}(x)$ is a compactly supported function of bounded variation and $u^{\varepsilon}(x, t)$ the unique admissible solution of the scalar convex law (4.1) with data (4.6). Then, the $L^{1}$ deviation between $u^{\varepsilon}$ and the two-term asymptotic solution $u_{w}^{\varepsilon}$ given by (4.7) in terms of the solution $\sigma$ of the Burgers equation (4.5) with data $u_{1}(x)$ is quadratically small, uniformly in time:

$$
\int_{-\infty}^{\infty}\left|u^{\varepsilon}(x, t)-u_{w}^{\varepsilon}(x, t)\right| d x \leqq \text { const } \varepsilon^{2},
$$

where the constant depends only on the diameter of the support of the perturbation $u_{1}$ and $f^{\prime \prime}\left(u_{0}\right)$.

Thus, for a genuinely nonlinear scale equation, we may legitimately write

$$
u^{\varepsilon}(x, t)=u_{0}+\varepsilon \sigma\left(x-\lambda_{0} t, \varepsilon t\right)+O\left(\varepsilon^{2}\right)
$$

if the error is interpreted in $L^{1}$.

One of the main reasons for anticipating the temporal uniformity of the expansion stems from the large-time decay to equilibrium in the total variation norm. For simplicity in the statements we shall henceforth restrict our attention to flux functions $f$ which are uniformly convex, i.e. $f^{\prime \prime}(u) \geqq \delta>0$. In general circumstances $\delta$ would be replaced by the minimum of $f^{\prime \prime}$ on the range of the solution in question. We first recall that for arbitrary $f$ the solution obeys a maximum principle in the total variation norm:

$$
T V u(\cdot, t) \leqq T V u_{0} .
$$

Second, the solution decays at the rate $t^{1 / 2}$ provided $f$ is strictly convex and the data $u_{0}$ are compactly supported: if $\left|u_{0}\right|_{\infty} \leqq M$, then

$$
T V u(\cdot, t) \leqq \operatorname{const}\left(T V u_{0} / t\right)^{1 / 2},
$$


where the constant depends only on $M$ and the diameter of the support of $u_{0}$ [1]. In the special case where $f$ is uniformly convex, i.e. $f^{\prime \prime}(u) \geqq \delta>0$ the constant depends only on $\delta$.

By interpolation, one obtains a general decay law of the form

$$
T V u(\cdot, t) \leqq \operatorname{const}\left(T V u_{0}\right)^{\alpha} / t^{\beta}
$$

for $0 \leqq \beta \leqq 1 / 2, \alpha+\beta=1$. We note that if one were to surmise a decay law with the structure (4.10) it would then follow from the group invariance (4.4) that $\alpha+\beta=1$. In the special case of Burgers equation (4.9) is an exact group invariance while for a general law it is an infinitesimal invariance. In any case, it is sufficient for the purposes of this section to appeal to a decay law of the form (4.10) where $\alpha$ and $\beta$ are arbitrary positive numbers such that $\alpha+\beta=1$.

Proof of Theorem 4.1. The approach is to compare the solution $u^{\varepsilon}$ of

$$
\partial_{t} u^{\varepsilon}+\partial_{x} Q f\left(u^{\varepsilon}, u_{0}\right)=\mu^{\varepsilon} \equiv-\partial_{x} c\left(u^{\varepsilon}, u_{0}\right)
$$

with the solution $u_{w}^{\varepsilon}$ of the truncated law

$$
\partial_{t} u_{w}^{\varepsilon}+\partial_{x} Q f\left(u_{w}^{\varepsilon}, u_{0}\right)=0 .
$$

Since both have the same initial data and both satisfy the entropy condition in the sense that they decrease from left to right across discontinuities, it follows from the stability corollary 3.1 that

$$
\int_{-\infty}^{\infty}\left|u^{\varepsilon}-u_{w}^{\varepsilon}\right|(t) d x \leqq\left|\mu^{\varepsilon}\right|\left(\mathscr{S}_{t}\right)
$$

where $\mathscr{S}_{t}$ denotes the strip $[0, t]$. In order to estimate the total mass of the cubic residual over the strip $\mathscr{S}_{t}$ we observe that

$$
\left|\mu^{\varepsilon}\right|\left(\mathscr{S}_{t}\right) \leqq \text { const } \int_{0}^{t}\left|u-u_{0}\right|^{2}(t) T V u(\cdot, t) d t
$$

as a consequence of the chain rule for measures, cf. Sect. 3. We have used the fact that

$$
\partial_{x} c\left(u, u_{0}\right)=q\left(u, u_{0}\right) \partial_{x} u=O\left(u-u_{0}\right)^{2} \partial_{x} u .
$$

The $L^{\infty}$ norm may be conveniently dominated by the total variation norm to yield a cubic bound of the form

$$
|\mu|\left(\mathscr{S}_{t}\right) \leqq \text { const } \int_{0}^{t} T V^{3} u(\cdot, s) d s .
$$

We estimate the right-hand side of the above inequality by using the maximum principle from (4.9) and the decay law in (4.10) as follows. For any $0<\tau \leqq T$, we have

$$
\int_{0}^{T}(T V u(\cdot, s))^{3} d s \leqq \mathrm{const} \int_{0}^{\tau} T V^{3} u_{0} d s+\mathrm{const} \int_{\tau}^{T}\left(T V u_{0}\right)^{3 / 2} s^{-3 / 2} d s .
$$

Since $T V u_{0}=\varepsilon C$, we choose $\tau=\min \left(T, \varepsilon^{-1}\right)$ to obtain $|\mu|\left(\mathscr{S}_{t}\right) \leqq C \varepsilon^{2}$, and this completes the proof. 


\section{The Asymptotic Theory for Systems}

In this section we shall establish the uniform temporal validity of weakly nonlinear asymptotics for systems of conservation laws

$$
\partial_{t} u+\partial_{x} f(u)=0
$$

which are strictly hyperbolic and genuinely nonlinear in the sense of Lax [14]. As in Sect. 4, the problem deals with the response of the solution operator to small amplitude perturbations of constant data,

$$
u(x, 0)=u_{0}+\varepsilon u_{1}(x)
$$

where $u_{1}$ is an arbitrary $\mathrm{BV}$ function with compact support. The general existence theorem of Glimm [6], discussed in Sect. 2 constructs a globally defined distribution solution of (5.1) using the random choice method with data (5.2) if $\varepsilon$ is sufficiently small. Our analysis will be restricted to these random choice solutions; the uniqueness problem for admissible solutions to conservation laws is currently an open problem in the general setting in which we are working.

The main assertion is that the spatial $L^{1}$-norm of the error term in the expansion

$$
u^{\varepsilon}(x, t)=u_{0}+\varepsilon \sum_{j=1}^{n} \sigma_{j}\left(\phi_{j}, \theta\right) r_{j}\left(u_{0}\right)+O\left(\varepsilon^{2}\right)
$$

is quadratically small, uniformly in time, cf. Theorem 5.1.

The proof is based upon uniform decay of the total variation norm together with refined estimates on the asymptotic rate of uncoupling of the characteristic fields with large time. For simplicity in the exposition we shall concentrate on systems of two equations for which the presence of Riemann invariants reduces several of the technicalities.

We shall begin with a brief review of the derivation of the generalized characteristic system for weak solutions of (5.1). We recall that in regime of smooth solutions, the conservative system (5.1) may be recast in characteristic form through the introduction of the left eigenvectors of $\nabla f: l_{j} \nabla f=\lambda_{j} l_{j}$. Multiplication of the quasilinear form,

$$
\partial_{t} u+\nabla f(u) \partial_{x} u=0
$$

by the left eigenvectors $l_{j}$ yields the canonical form

$$
l_{j} \cdot\left\{\partial_{t} u+\lambda_{j}(u) \partial_{x} u\right\}=0
$$

which reduces to diagonal form

$$
\partial_{t} w_{j}+\lambda_{k} \partial_{x} w_{j}=0, \quad k \neq j, \quad w_{j}=w_{j}(u(x, t)),
$$

in terms of the $j$-Riemann invariants $w_{j}$. The characteristic coordinates $w_{j}$ are usually introduced as solutions of the first order linear p.d.e.,

$$
r_{j}(u) \cdot \nabla w_{j}(u)=0,
$$

over the state space $R^{2}$ the diagonal form (5.4), for the composition of $w_{j}$ with a smooth solution $u=u(x, t)$, follows from the biorthogonality of left and right 
eigenvectors:

$$
l_{k} \cdot r_{j}=0, \quad k \neq j
$$

In view of (5.5), the mapping

$$
\left(u_{1}, u_{2}\right) \rightarrow\left(w_{1}, w_{2}\right)
$$

is locally invertible, leading to the common practice of writing the characteristic system in the form

$$
\partial_{t} w_{j}+\lambda_{k}\left(w_{1}, w_{2}\right) \partial_{k} w_{j}=0 ; \quad j=1,2, \quad k \neq j,
$$

for unknowns $w_{1}(x, t)$ and $w_{2}(x, t)$. Once (5.7) is solved, the original components are recovered by inverting the map (5.5).

Before discussing the generalized version of (5.7) for discontinuous solutions, we shall comment on the manner in which genuine nonlinearity is reflected in its structure. We recall that system (5.1) is genuinely nonlinear provided that the eigenvalues $\lambda_{j}$ are strictly monotone in the corresponding eigendirection:

$$
r_{j}(u) \cdot \nabla \lambda_{j}(u) \neq 0 .
$$

A simple computation shows that the first eigenderivative of $\lambda_{j}$ may be expressed as a second eigenderivative of $f$,

$$
r_{j} \nabla \lambda_{j}=l_{j} \cdot d^{2} f\left(r_{j}, r_{j}\right)
$$

and therefore that genuine nonlinearity means convexity in the eigendirections of a distinguished linear combination of the components of $f$. In the coordinate system of Riemann invariants, we have

$$
\frac{\partial}{\partial w_{j}}=r_{k} \cdot \nabla, \quad k \neq j
$$

and the condition of genuine nonlinearity becomes the monotonicity of $\lambda_{k}$ in the complementary direction:

$$
\frac{\partial}{\partial w_{j}} \lambda_{k}\left(w_{1}, w_{2}\right) \neq 0, \quad j \neq k
$$

For weak solutions in $\mathrm{BV} \cap L^{\infty}$, a generalized version of the classical characteristic system was introduced in [2] for the purpose of studying the large-time asymptotic behavior of the solution, in particular, for proving the Lax conjecture that the decay to $N$-waves of solutions to systems of conservation laws with compactly supported data. In [2] it was shown that if $u \in \mathrm{BV} \cap L^{\infty}$, then the conservative system (5.1) is equivalent to an inhomogeneous diagonal system of the form

$$
\partial_{t} w_{j}+\lambda_{k} \partial_{x} w_{j}=\mu_{j}
$$

where $\mu_{j}$ is a locally finite Borel measure concentrated on the shock set $\Gamma(u)$ of $u$. The derivative of (5.8) proceeds by rewriting the divergence form (5.1) in quasilinear form

$$
\partial_{t} u+\hat{\nabla} f(u) \partial_{x} u=0
$$


by applying the generalized chain rule for BV functions to the spatial gradient of the flux, cf. Sect. 2. Then one multiplies by the mean value,

$$
\hat{\nabla} w_{j}=\int_{0}^{1} \nabla w\left(s L_{v} u+(1-s) L_{-v} u\right) d s,
$$

of the $j$-Riemann invariant $w_{j}$, in order to obtain

$$
\begin{aligned}
\hat{\nabla} w_{j} \partial_{t} u+\hat{\nabla} w_{j} \nabla f \partial_{x} u & =0, \\
\hat{\nabla} w_{j} \partial_{t} u+\lambda_{k} \hat{\nabla} w_{j} \partial_{x} u & =\left\{\lambda_{k} \hat{\nabla} w_{j}-\hat{\nabla} w_{j} \nabla f\right\} \partial_{x} u .
\end{aligned}
$$

Since the chain rule implies that

$$
\partial_{t} w_{j}=\hat{\nabla} w_{j} \partial_{t} u ; \quad \partial_{x} w_{j}=\hat{\nabla} w_{j} \partial_{x} u,
$$

we arrive at the diagonal system

$$
\partial_{t} w_{j}+\lambda_{k} \partial_{x} w_{j}=\mu_{j} ; \quad \mu_{j} \equiv\left\{\lambda_{k} \hat{\nabla} w_{j}-\hat{\nabla} w_{j} \nabla f\right\} \partial_{x} u
$$

for the composite Riemann invariants $w_{j}=w_{j}(u(x, t))$ associated with a BV solution $u$. As always, the index $j$ and $k$ are distinct. The forcing measure $\mu_{j}$ is concentrated on the shock set $\Gamma$ of $u$ since the associated coefficient vanishes at $H_{1}$-almost all points of $\Gamma^{c}$ : if $(x, t) \in \Gamma^{c}(u)$, then the mean values reduce to pointwise values and we find

$$
\begin{gathered}
\hat{\nabla} w_{j}(x, t)=w_{j}(u(x, t)), \\
\lambda_{j} \hat{\nabla} w_{j}-\hat{\nabla} w_{j} \hat{\nabla} f=\lambda_{j} \nabla w_{j}-\nabla w_{j} \nabla f=0 .
\end{gathered}
$$

Remark. The technical derivation of the generalized characteristic system (5.9) is facilitated by replacing, at the very onset, a given $\mathrm{BV} \cap L^{\infty}$ solution by its symmetric mean,

$$
\bar{u}=\lim _{\varepsilon \rightarrow 0} u * \psi_{\varepsilon},
$$

obtained by mollifying with a standard radially symmetric approximation $\psi_{\varepsilon}$ of the $\delta$-function. It is easy to verify that, with the possible exception of a set with zero 1dimensional Hausdorff measure, $\bar{u}$ and $u$ coincide, $\Gamma(u)$ and $\Gamma(\bar{u})$ coincide and $\bar{u}$ assumes the average value $\bar{u}=\left(L_{v} u+L_{-v} u\right) / 2$ on the shock set. Henceforth we shall assume without loss of generality that $u=\bar{u}, H_{1}$ almost everywhere.

In the study of asymptotic behavior, there are a number of interesting consequences of the characteristic equations (5.9). We recall from [2] that the nonlinear modes of the system uncouple asymptotically at the rate $t^{-3 / 2}$ as a result of spatial total variation decay at the rate $t^{-1 / 2}$. Specifically, the solution generated by the random choice method applied to a genuinely nonlinear system of conservations with Cauchy data supported in a bounded interval I decays uniformly to equilibrium in the total variation norm at the same rate as a scalar solution:

$$
T V u(\cdot, t) \leqq \text { const }\left\{T V u_{0} / t\right\}^{1 / 2},
$$

where the constant depends only on $|I|$ and $f$, provided that the total variation of the initial data is sufficiently small.

We shall demonstrate next that the restriction of $\mu_{j}$ to a fixed time is cubic with 
respect to the total variation of $u$ at time $t$, in the sense that

$$
\left|\mu_{j}\right| \mathscr{S}\left(t_{1}, t_{2}\right) \leqq \text { const } \int_{t_{1}}^{t_{2}} T V^{3} u(\cdot, t) d t,
$$

for each strip between $t_{1}$ and $t_{2}$. The estimate (5.10) suggests that a midly coupled pair of scalar laws for Riemann invariants are governing the large-time behavior of the solution. The proof of (5.10) proceeds as follows. Since the forcing measure $\mu_{j}$ is concentrated on the shock set $\Gamma$, we may conveniently represent its action by appealing to the second form of Green's theorem to obtain

$$
\mu_{j}(B)=\int_{B} v_{t}\left[w_{j}\right]+\lambda_{k} v_{x}\left[w_{j}\right] d H_{1}
$$

for all Borel subsets $B \subset \Gamma$ cf. Sect. 2. We assert that the integrand is cubic in the sense that

$$
\left|v_{t}\left[w_{j}\right]+\lambda_{k} v_{x}\left[w_{j}\right]\right| \leqq \text { const }|[u]|^{3} .
$$

Now, across a $j$-shock, the change in a $j$-Riemann invariant is third order with respect to the magnitude of the shock, so that (5.12) is obvious. Across a $k$-shock, $k \neq j$, the change in a $j$-Riemann invariant is first order while the speed of propagation $s$ coincides with the average characteristic speed to second order [15]:

$$
s \equiv-v_{t} / v_{x}=\left\{\lambda_{k}\left(u^{+}\right)+\lambda_{k}\left(u^{-}\right)\right\} / 2+O\left(u^{+}-u^{-}\right)^{2} .
$$

Since, for any smooth function $g$, the mean value of $g$ coincides with $g$ of the mean value to second order,

$$
g\left(\frac{u^{+}+u^{-}}{2}\right)=\frac{g\left(u^{+}\right)+g\left(u^{-}\right)}{2}+O\left(u^{+}-u^{-}\right)^{2},
$$

it follows that at each jump point $p$ (corresponding to a $k$-shock), we have

$$
\begin{gathered}
s=\lambda_{k}(u)+O\left(u^{+}-u^{-}\right)^{2}, \quad u=\frac{u^{+}+u^{-}}{2}, \\
v_{t}\left[w_{j}\right]+v_{x} \lambda_{k}\left[w_{j}\right]=-v_{x}\left[s-\lambda_{k}\right]\left[w_{j}\right]=O\left(u^{+}-u^{-}\right)^{3} .
\end{gathered}
$$

The second stage in the analysis of the asymptotic uncoupling of the characteristic coordinates $w_{j}$ is to extract the Burgers' operators from the left-hand side of the generalized system (5.9) in order to produce a purely diagonal "principal part" responding to an asymptotically small inhomogeneous term $\beta_{j}$ :

$$
\partial_{t} w_{j}+\lambda_{k}^{0} \partial_{x} w_{j}=\beta_{j}, \quad \lambda_{1}^{0}=\lambda_{1}\left(w_{1}, w_{2}^{0}\right), \quad \lambda_{2}^{0}=\lambda_{2}\left(w_{1}^{0}, w_{2}\right)
$$

where $w_{j}^{0}$ denotes the value of $j$-Riemann invariant at the unperturbed state $u_{0}$. The structure (5.13) is obtained by rewriting (5.9) in the form

$$
\begin{aligned}
& \partial_{t} w_{1}+\lambda_{2}\left(w_{1}, w_{2}^{0}\right) \partial_{x} w_{1}=\mu_{1}+\left\{\lambda_{2}\left(w_{1}, w_{2}^{0}\right)-\lambda_{2}\left(w_{1}, w_{2}\right)\right\} \partial_{x} w_{1}, \\
& \partial_{t} w_{2}+\lambda_{1}\left(w_{1}^{0}, w_{2}\right) \partial_{x} w_{2}=\mu_{2}+\left\{\lambda_{1}\left(w_{1}^{0}, w_{2}\right)-\lambda_{1}\left(w_{1}, w_{2}\right)\right\} \partial_{x} w_{2},
\end{aligned}
$$

so that $\beta_{j}$ consists of two contributions, $\beta_{j}=\mu_{j}+\mu_{j}^{\prime}$ where

$$
\begin{aligned}
& \mu_{1}^{\prime}=\left\{\lambda_{2}\left(w_{1}, w_{2}^{0}\right)-\lambda_{2}\left(w_{1}, w_{2}\right)\right\} \partial_{x} w_{1} \\
& \mu_{2}^{\prime}=\left\{\lambda_{1}\left(w_{1}^{0}, w_{2}\right)-\lambda_{1}\left(w_{1}, w_{2}\right)\right\} \partial_{x} w_{2} .
\end{aligned}
$$


In general, the measure $\beta_{j}$ is not concentrated on the shock set of $u$, but it has the same asymptotic rate of decay as the total mass of the basic forcing measure $\mu_{j}$ :

$$
\begin{aligned}
\left|\beta_{j}\right| \mathscr{S}\left(t_{1}, t_{2}\right) \leqq & \text { const } \int_{t_{1}}^{t_{2}} T V^{3} u(\cdot, t) d t \\
& + \text { const } \int_{t_{1}}^{t_{2}} T V^{2} u(\cdot, \pi t) T V u(\cdot, t) d t
\end{aligned}
$$

for an appropriate delay parameter $\pi, 0<\pi<1$. Thus the restriction of $\beta_{j}$ to a fixed line $t=\tau$ decays like $\tau^{-3 / 2}$. The proof of the bound (5.14) depends upon a refined estimate dealing with decay rates of major and minor waves in each of the characteristic fields. We shall return to this point below.

The final stage in the asymptotic analysis of the coordinates $w_{j}$ is to formulate an inhomogeneous pair of conservative scalar equations to which the stability theorems of Sect. 2 may be applied to yield the desired approximation theorem for weakly nonlinear asymptotic in the $L^{1}$ norm. Let us introduce flux functions $\Lambda_{k}$ as the primitives of the background characteristic speeds:

$$
\begin{aligned}
& \Lambda_{1}^{\prime}(z)=\lambda_{1}\left(w_{1}^{0}, z\right), \\
& \Lambda_{2}^{\prime}(z)=\lambda_{2}\left(z, w_{2}^{0}\right),
\end{aligned}
$$

where the dependence of $\Lambda_{k}$ on $\left(w_{1}^{0}, w_{2}^{0}\right)$ is suppressed for simplicity. We then arrive at the conservative inhomogeneous system

where

$$
\partial_{t} w_{j}+\partial_{x} \Lambda_{k}\left(w_{j}\right)=\widetilde{\beta}_{j}
$$

$$
\begin{aligned}
& \widetilde{\beta}_{1}=\beta_{1}+\beta_{1}^{\prime} \equiv \beta_{1}+\left\{\partial_{x} \Lambda_{2}\left(w_{1}\right)-\lambda_{2}\left(w_{1}, w_{2}^{0}\right) \partial_{x} w_{1}\right\}, \\
& \widetilde{\beta}_{2}=\beta_{2}+\beta_{2}^{\prime} \equiv \beta_{2}+\left\{\partial_{x} \Lambda_{1}\left(w_{2}\right)-\lambda_{1}\left(w_{1}^{0}, w_{2}\right) \partial_{x} w_{2}\right\} .
\end{aligned}
$$

It is easily seen that the forcing measure $\widetilde{\beta}_{j}$ satisfies the same decay estimate (5.14) as $\beta_{j}$. For example, the term

$$
\beta_{1}^{\prime}=\left\{\hat{\Lambda}_{2}^{\prime}\left(w_{1}\right)-\lambda_{2}\left(w_{1}, w_{2}\right)\right\} \partial_{x} w_{1}
$$

is concentrated on the shock set $\Gamma$ as a consequence of the definition (5.14) and the chain rule. On the shock set it admits a quadratic coefficient

$$
\hat{\Lambda}_{2}^{\prime}\left(w_{1}\right)-\lambda_{2}\left(w_{1}, w_{2}^{0}\right)=O\left(u^{+}-u^{-}\right)^{2}
$$

from which the estimate

$$
\left|\beta_{1}^{\prime}\right| \mathscr{S}\left(t_{1}, t_{2}\right) \leqq \mathrm{const} \int_{t_{1}}^{t_{2}} T V^{3} u(\cdot, t) d t
$$

follows easily. An identical argument works for $\beta_{2}^{\prime}$ using (5.16) and the chain rule. The main observation for both cases is simply that the mean of $g$ differs from $g$ of the mean by second order terms.

We have thus obtained a structure where each of the invariants satisfies a scalar law with rapidly decaying forcing term from which the main result on the uniform validity of weakly nonlinear asymptotics may be deduced.

Theorem 5.1. Consider a random choice solution $u^{\varepsilon}$ of the Cauchy problem for a 
strictly hyperbolic genuinely nonlinear system of two conservative laws with initial data of the form

$$
u^{\varepsilon}(x, 0)=u_{0}+\varepsilon \sum_{j=1}^{2} \sigma_{j}^{0}(x) r_{j}\left(u_{0}\right),
$$

where $\sigma_{j}^{0}$ are arbitrary compactly supported functions of bounded variation. Then the formal weakly nonlinear asymptotic solution

$$
u_{w}^{\varepsilon}(x, t) \equiv u_{0}+\varepsilon \sum_{j=1}^{2} \sigma_{j}\left(\phi_{j}, \tau\right) r_{j}\left(u_{0}\right)
$$

whose profile is modulated along linearized characteristics $\phi_{j} \equiv x-\lambda_{j}\left(u_{0}\right) t$, in accordance with Burgers equations

$$
\partial_{\tau} \sigma_{j}+\frac{1}{2} \partial_{\phi}\left(b_{j} \sigma_{\phi}^{2}\right)=0, \quad b_{j}=l_{j} \nabla^{2} f\left(r_{j}, r_{j}\right)\left(u_{0}\right),
$$

satisfies a uniform estimate

$$
\int_{-\infty}^{\infty}\left|u^{\varepsilon}-u_{w}^{\varepsilon}\right|(t) d t \leqq \text { const } \varepsilon^{2}
$$

where the constant depends only on $f$ and the diameter of the support of the perturbations $\sigma_{j}^{0}$.

Proof. Since the mapping $\left(u_{1}, u_{2}\right) \rightarrow\left(w_{1}, w_{2}\right)$ is locally invertible it suffices to estimate the $L^{1}$ difference between the composition of the Riemann invariants $w_{j}$ with the exact solution $u^{\varepsilon}$ and the approximate solution $u_{w}^{\varepsilon}$. Now, as a consequence of the uniform decay law

$$
T V u(\cdot, t) \leqq \text { const }\left\{T V u_{0} / t\right\}^{1 / 2}
$$

for compactly supported solutions, it follows that the total mass of the forcing measure $\widetilde{\beta}_{j}$ is quadratic in $\varepsilon$,

$$
\left|\widetilde{\beta}_{j}\right| \mathscr{S}(0, t) \leqq \text { const } \varepsilon^{2},
$$

in a fashion similar to the scalar case. Thus the function $w_{j}$ satisfies a scalar law (5.17) for which the right-hand side is quadratically small. We may therefore apply the stability theorems of Sect. 3 if $w_{j}$ satisfies the entropy condition, stating that the left limit exceeds the right limit across shocks. For the moment let us assume the entropy condition and proceed.

The function $w_{j}$ is uniformly approximated by a multiple-scale expansion.

$$
w_{j}^{\varepsilon} \equiv w_{j}\left(u_{0}\right)+\varepsilon \sigma_{j}\left(\phi_{j}, \tau\right),
$$

where $\phi_{j}=x-\lambda_{j}\left(u_{0}\right) t, \tau=\varepsilon t$ and $\sigma_{j}$ satisfies the Burgers equation

$$
\partial_{\tau} \sigma_{j}+\frac{1}{2} \partial_{x}\left(b_{k} \sigma_{j}^{2}\right)=0 \text {. }
$$

As we remarked above, a simple calculation shows that

$$
b_{k}=\Lambda_{k}^{\prime \prime}\left(w_{j}^{0}\right)=\frac{\partial \lambda_{j}}{\partial w_{k}}\left(w_{1}^{0}, w_{2}^{0}\right)=l_{k}^{2} \cdot d^{2} f\left(r_{k}, r_{k}\right)\left(u_{0}\right) \text {. }
$$

The initial data for (5.18) are determined as follows. Write the data for the Riemann 
invariants $w_{j}$ in the form of a compactly supported perturbation of a constant state:

$$
\begin{aligned}
& w_{j}^{\varepsilon}(x, 0)=w_{j}\left(u^{\varepsilon}(x, 0)\right), \\
& w_{j}^{\varepsilon}(x, 0)=w_{j}\left(u^{0}\right)+\varepsilon w_{j}^{1}(x)+O\left(\varepsilon^{2}\right),
\end{aligned}
$$

where $O\left(\varepsilon^{2}\right)$ and $w_{j}^{1}$ vanish outside a bounded interval. Take the initial value of the structure function $\sigma_{j}$ to be $\sigma_{j}(x, 0)=w_{j}^{1}(x)$. It then follows from the stability results of Sect. 3 that the solution of (5.18) with truncated data $w_{j}\left(u^{0}\right)+\varepsilon w_{j}^{1}(x)$, and the solution of (5.18) with exact data (5.19) differ in $L^{1}$ by a quantity $O\left(\varepsilon^{2}\right)$ uniformly in time. The former solution is the weakly nonlinear asymptotic solution $w_{j}^{\varepsilon}$ and we obtain the desired result

$$
\int_{-\infty}^{\infty}\left|w_{j}^{\varepsilon}-w_{j}\left(u_{0}\right)-\varepsilon \sigma_{j}\left(\phi_{j}, \tau\right)\right| d x \leqq \text { const } \varepsilon^{2}
$$

Several normalizations remain to be discussed in conjunction with the entropy condition. It is standard to normalize the direction of genuinely nonlinear eigenvectors by the condition

$$
r_{j} \cdot \nabla \lambda_{j}>0
$$

and to normalize the direction of the gradient of the corresponding Riemann invariant by the condition

$$
r_{j} \cdot \nabla w_{k}>0, \quad k \neq j .
$$

Under these normalizations, the entropy condition for weak shocks in a $2 \times 2$ system requires that, across a $j$-shock, the major Riemann invariant $w_{k}, k \neq j$ decreases from left to right. The change in the minor invariant $w_{j}$ across a $j$-shock is third order and adopts a sign depending on the state and system in question. It turns out that for the isentropic equations of gas dynamics for a polytropic gas, both invariants decrease from left to right across an admissible shock, so that the entropy condition for Riemann invariants, viewed as solutions to scalar inhomogeneous laws is automatically satisfied. More generally, one may assert that for any system of two equations satisfying the Glimm-Lax interaction condition (that two $k$-shocks interact to produce a $k$-shock and a $j$-rarefaction wave, $j \neq k$ ) admissible shocks are those for which both Riemann invariants decrease from left to right. Since the random choice method generates admissible solutions [15], the proof is complete for the aforementioned broad class of systems once we remark that the flux functions $\Lambda_{k}$ are convex due to the identity (5.19) and the normalizations (5.21), (5.22).

An additional remark is required to complete the proof for systems which do not satisfy the Glimm-Lax interaction condition. It is a straightforward corollary of Theorem 3.1 that the $L^{1}$ deviation between BV solutions $u_{1}$ and $u_{2}$ to inhomogeneous scalar laws can be estimated from above by the total mass of the forcing measures $\mu_{j}$ and the total variation of those shocks which violate the entropy condition

Proposition. If $u_{j} \in \mathrm{BV} \cap L^{\infty}\left(R^{2}\right)$ then

$$
\int_{-\infty}^{\infty}\left|u_{1}-u_{2}\right|(t) d x \leqq \int_{-\infty}^{\infty}\left|u_{1}-u_{2}\right|(0)+2 \sum\left|\mu_{j}\right|(\mathscr{S})+\sum\left|\partial_{x} u_{j}\right|(\mathscr{S} \cap N)
$$

where $\mathscr{S}$ denotes the strip $[0, t]$ and $N=\{p \in \Gamma(u):[u](p)<0\}$. 
For general systems we need only remark that the total strength of all waves which violate the entropy, when viewed from a specific Riemann invariant, is cubic in the total variation of the solution $u$, since the change in a minor invariance across a shock is third order in the shock strength. Hence the violation produces an error on exactly the same order as the forcing measure $\widetilde{\beta}_{j}$ itself, namely $t^{-3 / 2}$.

The final detail in the proof concerns the verification of the decay estimate (5.14) for total mass of $\beta_{j}$. As a preliminary remark in this connection, we observe that the structure (5.13a) immediately yields a quadratic estimate since the coefficients are themselves first order:

$$
\begin{aligned}
& \left|\lambda_{2}\left(w_{1}, w_{2}^{0}\right)-\lambda_{2}\left(w_{1}, w_{2}\right)\right| \leqq\left|w_{2}-w_{2}^{0}\right|_{\infty} \leqq \text { const } T V u, \\
& \left|\lambda_{1}\left(w_{1}^{0}, w_{2}\right)-\lambda_{1}\left(w_{1}, w_{2}\right)\right| \leqq\left|w_{1}-w_{1}^{0}\right|_{\infty} \leqq \text { const } T V u .
\end{aligned}
$$

We therefore have a crude estimate

$$
\left|\mu_{j}^{\prime}\right| \mathscr{S}\left(t_{1}, t_{2}\right) \leqq \mathrm{const} \int_{t_{1}}^{t_{2}} T V^{2} u(\cdot, t) d t .
$$

In order to see how the cubic form (5.14) arises let us assume without loss of generality that

$$
\lambda_{1}\left(w_{1}^{0}, w_{2}^{0}\right)<0<\lambda_{2}\left(w_{1}^{0}, w_{2}^{0}\right),
$$

and consequently that

$$
\lambda_{1}\left(w_{1}, w_{2}\right) \leqq-\delta<\delta \leqq \lambda_{2}\left(w_{1}, w_{2}\right)
$$

for small amplitude solutions $w_{j}=w_{j}(u(x, t))$. In this situation, initial data with compact support generates a wave pattern where eventually most of the 1 -waves lie in the left-hand space $x>0$ while most of the 2-waves lie in the right half-space $x<0$. Indeed it can be shown that waves in the complementary half-spaces satisfy

$$
\begin{aligned}
& \underset{x \leq 0}{T V} w_{1}(\cdot, t) \leqq \text { const } T V^{2} u(\cdot, \pi t)+\text { const } T V^{3} u(\cdot, t), \\
& \underset{x \geq 0}{T V} w_{2}(\cdot, t) \leqq \text { const } T V^{2} u(\cdot, \pi t)+\text { const } T V^{3} u(\cdot, t),
\end{aligned}
$$

for an appropriate delay parameter $\pi$, depending on the size of the support of the initial data. The upper bounds (5.23) and (5.24) imply the desired estimate (5.14). Since estimates of the type (5.23) and (5.24) are discussed in some detail in [2] and [18] we shall only give a brief indication of the proof.

Consider (5.23), fix $t_{0}$ and let $x_{b}(t)$ denote the background generalized 2characteristic through $\left(0, t_{0}\right)$. Since the speed of propagation is strictly positive, this characteristic exits the left edge of the support of $u$ at a time $t^{*} \geqq \pi t_{0}$ for some $\pi, 0<\pi<1$. Consider the closed "triangular" region $\Omega$ bounded on the top by

$$
C_{t}=\left\{(x, t): x_{l}(t) \leqq x \leqq 0\right\},
$$

and on the left and right sides by

$$
\begin{aligned}
& C_{l}=\left\{\left(x_{l}(t), t\right): t^{*} \leqq t \leqq t_{0}\right\}, \\
& C_{r}=\left\{\left(x_{b}(t), t\right): t^{*} \leqq t \leqq t_{0}\right\},
\end{aligned}
$$


where $x_{l}$ denotes the left edge of the support of $u$. By construction, $\Omega$ has no incoming 1 -waves. The only 1 -waves which cross the boundary of $\Omega$ are those which leave through the horizontal top segment $C_{T}$. Now, for a general region, it is known for random choice solutions, that the total amount of outgoing $j$-waves equals the total amount of incoming $j$-waves modulo a quantity which is on the order of the square of the total variation of the solution restricted to the domain of dependence of the region which is here contained in the line $t=\tau t_{0}$. Since there are no incoming 2waves for $\Omega$, the total amount of outgoing 2 -waves is of the order $T V^{2} u\left(\cdot \pi t_{0}\right)$. Since the change in $w_{1}$ across 1 -waves is cubic we arrive at the estimate

$$
\underset{x \leqq 0}{T V} w_{1}(\cdot, t) \leqq \text { const } T V^{2} u(\cdot, \pi t)+\text { const } T V^{3} u(\cdot, t) .
$$

\section{Weakly Nonlinear Asymptotics for Periodic Solutions}

This section is concerned with the validity of weakly nonlinear expansions in the setting of the Cauchy problem with periodic data. We shall first discuss genuinely nonlinear scalar equations

$$
\partial_{t} u+\partial_{x} f(u)=0, \quad f^{\prime \prime}>0,
$$

and establish the uniform temporal validity of the first two terms of the classical expansion.

Theorem 6.1. Suppose that $u_{1}(x)$ is an arbitrary periodic function of bounded variation with period $p$ and that $u^{\varepsilon}(x, t)$ is the unique admissible solution of the convex scalar law (6.1) with perturbed data

$$
u^{\varepsilon}(x, 0)=u_{0}+\varepsilon u_{1}(x),
$$

where $u_{0}$ is a constant. Then, the $L^{1}$ deviation per period between $u^{\varepsilon}$ and the two-term expansion

$$
u_{w}^{\varepsilon} \equiv u_{0}+\varepsilon \sigma\left(x-\lambda_{0} t, \varepsilon t\right)
$$

determined by the unique periodic solution $\sigma$ of Burgers equation

$$
\partial_{t} \sigma+b \partial_{x} \sigma^{2}=0, \quad b=f^{\prime \prime}\left(u_{0}\right) / 2
$$

with data $u_{1}(x)$, is cubically small uniformly in time:

$$
\int_{a}^{a+p}\left|u^{\varepsilon}(x, t)-u_{w}^{\varepsilon}(x, t)\right| d x \leqq \text { const } \varepsilon^{3},
$$

where the constant depends only on the period $p$ and the $L^{\infty}$ norm of the structure function $u_{1}$.

Proof. The demonstration follows the lines of the case of compactly supported perturbations. In the periodic case, the total variation is computed over a period interval

$$
T V_{p} u=T V\{u(\cdot, t): a \leqq x \leqq a+p\} .
$$

For the periodic case we have both the estimate from the classical maximum 
principle,

$$
T V_{p} u(\cdot, t) \leqq T V_{p} u(\cdot, 0)
$$

and the well known strong decay estimate $([1,14])$,

$$
T V_{p}(\cdot, t) \leqq \text { const }|u(\cdot, 0)|_{L^{1}} / t
$$

obtained from studying the spreading of characteristics. One can estimate the $L^{1}$ norm per period by the product of the period and the total variation norm, $|u|_{L^{1}} \leqq p T V_{p} u$, and the above three facts yield the estimate

$$
T V_{p} u(\cdot, t) \leqq \text { const } T V_{p} u_{0} / 1+t \text {. }
$$

We remark in passing that the relevant constants actually depend only on the minimum value of $f^{\prime \prime}$ over the smallest closed interval containing the range of the initial data; in particular, if $f$ is uniformly convex, $f^{\prime \prime}>\delta>0$ the constants depend only on $\delta$.

In the presence of the decay estimate in (6.4) one may establish the uniform cubic bound in (5.3) by restricting attention to a period interval $[a, a+p]$, where $a$ is arbitrary and repeating the steps in the proof of Theorem 4.1 for compactly supported perturbations. We only need to remark that immediate extensions of Theorem 3.1 and Corollary 3.1 are valid for periodic functions. Specifically, if $u_{1}$ and $u_{2}$ are arbitrary functions in $\mathrm{BV} \cap L^{\infty}$ with spatial periodicity $p$, then the following uniform $L^{1}$ stability estimate holds:

$$
\begin{aligned}
\int_{a}^{a+p}\left|u_{1}(x, t)-u_{2}(x, t)\right| d x \leqq & \int_{a}^{a+p}\left|u_{1}(x, \tau)-u_{2}(x, \tau)\right| d x \\
& +\sum_{j} \theta_{j}\left(\mathscr{S}_{p}\right)+\sum_{j}\left|\mu_{j}\right|\left(\mathscr{S}_{p}\right),
\end{aligned}
$$

where $\mathscr{S}_{p}$ denotes the intersection of the closed temporal strip $[\tau, t]$ with the spatial period interval $[a, a+p]$. Here $f$ is an arbitrary smooth function and the measure $\mu_{j}$ and $\theta_{j}$ are defined exactly as in Sect. 3:

$$
\begin{aligned}
\mu_{j} & =\partial_{t} u_{j}+\partial_{x} f\left(u_{j}\right), \\
\theta_{j}(E) & =\int_{E \cap \Gamma\left(u_{j}\right)} \tau\left(v, u_{j}\right) d H_{1} .
\end{aligned}
$$

In the special case where $f$ is strictly convex and $u_{j}$ is admissible in the sense that $u_{j}^{-}>u_{j}^{+}$at $H^{1}$-almost all points of its shock set $\Gamma\left(u_{j}\right)$, one easily obtains the analogue of Corollary 3.1, namely

$$
\int_{a}^{a+p}\left|u_{1}(x, t)-u_{2}(x, t)\right| d x \leqq \int_{a}^{a+p}\left|u_{1}(x, \tau)-u_{2}(x, \tau)\right| d x+2 \sum_{j}\left|\mu_{j}\right|\left(\mathscr{S}_{p}\right)
$$

With the aid of the stability estimate (6.6), one may regard $u^{\varepsilon}$ as a solution of the inhomogeneous equation

$$
\partial_{t} u^{\varepsilon}+\partial_{x} Q f\left(u^{\varepsilon}, u_{0}\right)=\mu^{\varepsilon} \equiv-\partial_{x} c\left(u^{\varepsilon}, u_{0}\right)
$$

and compare it with the solution $u_{w}^{\varepsilon}$ of the quadratically truncated homogeneous law

$$
\partial_{t} u_{w}+\partial_{x} Q f\left(u_{w}^{\varepsilon}, u_{0}\right)=0
$$


Here we have the stronger estimate

$$
\begin{aligned}
\left|\mu^{\varepsilon}\left(\mathscr{S}_{p}\right)\right| & \leqq \text { const } \int_{0}^{T}\left|T V_{p} u^{\varepsilon}(\cdot, s)\right|^{3} d s \\
& \leqq \operatorname{const}\left(T V_{p} u^{\varepsilon}(\cdot, 0)\right)^{3} \int_{0}^{T}(1+s)^{-3} d s \\
& \leqq \operatorname{const} \varepsilon^{3}
\end{aligned}
$$

by using the faster decay law from (5.4). This completes the proof.

We shall next discuss the response of the solution operator of a genuinely nonlinear system of two conservation laws to initial data in the form of a periodic perturbation of a constant state.

Theorem 6.2. Consider a random choice solution $u^{\varepsilon}$ to the Cauchy problem for a system of two genuinely nonlinear equations with initial data of the form

$$
u^{\varepsilon}(x, 0)=u_{0}+\varepsilon \sum_{j=1}^{2} \sigma_{j}^{0}(x) r_{j}\left(u_{0}\right)
$$

where $\sigma_{j}^{0}$ are arbitrary periodic functions of bounded variation with period $p$. Then, the asymptotic solution

$$
u_{w}^{\varepsilon}=u_{0}+\varepsilon \sum_{j=1}^{2} \sigma_{j}(\phi, \tau) r_{j}\left(u_{0}\right)
$$

determined by two Burgers equations

$$
\begin{gathered}
\partial_{\tau} \sigma_{j}+\frac{1}{2} b_{j} \partial_{\phi} \sigma_{j}^{2}=0, \quad j=1,2, \\
b_{j} \equiv l_{j} \nabla^{2} f\left(r_{j}, r_{j}\right)\left(u_{0}\right)
\end{gathered}
$$

along linearized characteristics $\phi=x-\lambda_{j}\left(u_{0}\right)$ t, satisfies a uniform estimate

$$
\int_{a}^{a+p}\left|u^{\varepsilon}-u_{w}^{\varepsilon}\right|(t) d x \leqq \text { const } \varepsilon^{2} T
$$

in the interval $0 \leqq t \leqq T$. The constant depends only on $f$ and $T$ is arbitrary.

Several remarks are appropriate concerning the interpretation of the stability estimate (6.7) in view of the decay laws for periodic solutions. For a genuinely nonlinear system, the random choice solution to the periodic Cauchy problem with small data satisfies the following two sharp estimates:

$$
T V_{p} u(\cdot, t) \leqq \text { const } T V_{p} u(x, 0)
$$

if $T V_{p} u_{0}$ is sufficiently small, and

$$
T V_{p} u(\cdot, t) \leqq \text { const } / t
$$

if $|u(x, 0)|_{\infty}$ is sufficiently small. The constants depend only on $f$ and the point in state space in the neighbourhood of which the analysis takes place. We shall show below that the bound (6.8) in conjunction with the generalized characteristic system leads directly to the quadratic $L^{1}$ bound (6.7). The additional information contained in the decay law (6.9) is not required for the proof of (6.7). 
As a corollary, one deduces that the $L^{1}$ deviation per period satisfies

$$
\int_{a}^{a+p}\left|u^{\varepsilon}-u_{w}^{\varepsilon}\right|(t) d t \leqq \text { const } \varepsilon^{1+r}
$$

in the interval of time $0 \leqq t \leqq T=1 / \varepsilon^{1-r}$ of $0 \leqq r \leqq 1$. The estimate is meaningful if $r>0$, since each of the terms of the integrand has order $\varepsilon$ in the interval $0 \leqq t \leqq 1 / \varepsilon$.

A question arises regarding the optimality of the bound at times of order $1 / \varepsilon$. It appears that phase shifts induced by the nonlinear structure of the eigenvalues of a system will preclude agreement of $u^{\varepsilon}$ and $u_{w}^{\varepsilon}$ beyond first order at time $1 / \varepsilon$. We shall argue that for genuinely nonlinear systems with periodic data of amplitude $\varepsilon$, the wave form retains the order $\varepsilon$ in an interval of time of order $1 / \varepsilon$. Thus in general both the exact solution and the approximate asymptotic solution will be of order $\varepsilon$ at times of $1 / \varepsilon$, with discrepancies in wave location which lead to a global first order error:

$$
\int_{a}^{a+p}\left|u^{\varepsilon}\left(, \frac{1}{\varepsilon}\right)-u_{w}^{\varepsilon}\left(, \frac{1}{\varepsilon}\right)\right| d t \simeq \text { const } \varepsilon .
$$

The supporting evidence for the argument that (6.7) is sharp at time $T=1 / \varepsilon$ is the following. We recall that for a uniformly convex scalar law

$$
\partial_{t} u+\partial_{x} f(u)=0, \quad f^{\prime \prime}(u) \geqq \delta>0,
$$

the temporal decay profile of a periodic solution $u(x, t)$ with period $p$ is independent of the pointwise amplitude of the data in the sense that

$$
|u(\cdot, t)-\bar{u}|_{\infty} \leqq 1 / \delta t
$$

while

$$
\lim _{t \rightarrow \infty} t|u(\cdot, t)-\bar{u}|_{\infty}=f^{\prime \prime}(\bar{u}),
$$

see [14]. Here $\bar{u}$ denotes the time-invariant mean of the solution:

$$
\bar{u} \equiv \frac{1}{p} \int_{0}^{p} u(x, 0) d x=\frac{1}{p} \int_{0}^{p} u(x, t) d x .
$$

We observe that the right-hand sides of (6.11) and (6.12) are independent of the amplitude of the initial data.

One of the striking features of the temporal evolution is that the size of the initial data influences only the time at which the decay law (6.11) becomes accurate. The smaller the amplitude of the data the longer the elapsed time before (6.11) becomes sharp; the larger the amplitude of the data the shorter the elapsed time.

We shall illustrate this feature with an explicit solution of Burgers equation, $\partial_{t} u+\partial_{x}\left(u^{2} / 2\right)=0$, with piecewise constant periodic data consisting of two constant states:

$$
\begin{gathered}
u(x, 0)=\left\{\begin{array}{r}
-b \text { if } 0<x<1 \\
b \text { if } 1<x<2
\end{array}\right\}, \quad b>0 . \\
u(x+2,0)=u(x, 0) .
\end{gathered}
$$


The jump discontinuities located at the even integers generate centered rarefaction waves while the jump discontinuities at the odd integers generate stationary shock waves. The resulting solution $u(x, t)$ presents a pattern of decaying (centered) rarefaction waves separated by decaying (stationary) shock waves. In the period interval $-1 \leqq x \leqq 1$ the solution is expressed explicitly in form

$$
\begin{aligned}
& u(x, t)=x / t \quad \text { if } \quad-b \leqq x / t \leqq b \\
& =-b \quad \text { if } \quad x / t \leqq-b \\
& =b \quad \text { if } \quad x / t \geqq b \text {, }
\end{aligned}
$$

representing a centered rarefaction wave. At each time $t$ the strength $s$ of the stationary shocks,

$$
s \equiv \lim _{\beta \rightarrow 0}\{u(n+\beta, t)-u(n-\beta, t)\}, \quad n=\text { odd integer, }
$$

is independent of the amplitude $b$ if $t \leqq 1 / b$, i.e.

$$
s=\frac{2}{t}, \quad \text { if } \quad t \geqq 1 / b .
$$

In other words, oscillating data of amplitude $b$ generates a solution with amplitude $\theta b$ at time $\theta / b$ :

$$
|u(\cdot, \theta / b)|_{\infty}=\theta|u(\cdot, 0)|_{\infty} .
$$

The only feature which the number $b$ influences is the time at which the rarefaction waves begin to interact with the shocks: the smaller the value of $b$ the larger the elapsed time before interaction begins.

In the setting of genuinely nonlinear systems, one may anticipate a similar result: for a broad class of periodic data with amplitude $\varepsilon$, the wave form retains the order $\varepsilon$ in an interval of time of order $1 / \varepsilon$ :

$$
\left|u^{\varepsilon}\left(\cdot, \frac{\theta}{\varepsilon}\right)\right|_{\infty} \cong \operatorname{const} \theta\left|u^{\varepsilon}(\cdot, 0)\right|_{\infty} .
$$

Thus, in general both the exact solution and the approximate asymptotic solution with periodic data of order $\varepsilon$ will contain waves of order $\varepsilon$ and times of order $1 / \varepsilon$. Phase shifts induced by the nonlinear interactions of waves indifferent characteristics fields will most likely preclude agreement of $u^{\varepsilon}$ and $u_{w}^{\varepsilon}$ beyond first order at time $1 / \varepsilon$. It is only for a scalar law (governing a single nonlinear channel) that phase errors are absent, allowing temporally uniform agreement of order $\varepsilon^{2}$.

Proof of Theorem 6.2. The main step in the demonstration is an analysis of the generalized characteristic system

$$
\partial_{t} w_{j}+\lambda_{k} \partial_{x} w_{j}=\mu_{j}
$$

for the Riemann invariants $w_{j}$. As in the case of compactly supported perturbations one is lead to rewrite system (6.13) in the form of two coupled scalar equations

$$
\begin{aligned}
& \partial_{t} w_{1}+\lambda_{2}\left(w_{1}, w_{2}^{0}\right) \partial_{x} w_{1}=\mu_{1}+\left\{\lambda_{2}\left(w_{1}, w_{2}^{0}\right)-\lambda_{2}\left(w_{1}, w_{2}\right)\right\} \partial_{x} w_{1}, \\
& \partial_{t} w_{2}+\lambda_{1}\left(w_{1}^{0}, w_{2}\right) \partial_{x} w_{2}=\mu_{2}+\left\{\lambda_{1}\left(w_{1}^{0}, w_{2}\right)-\lambda_{1}\left(w_{1}, w_{2}\right)\right\} \partial_{x} w_{2} .
\end{aligned}
$$


The cubic character of the measures $\mu_{j}$ concentrated on the shock set leads directly to an estimate of the form

$$
\left|\mu_{j}\right|\left\{S_{p}(T)\right\} \leqq \text { const } \varepsilon^{2}
$$

by appealing to the decay estimate

$$
T V_{p} u(\cdot, t) \leqq \operatorname{const}\left(T V_{p} u_{0}\right)^{\alpha} / t^{\beta} ; \quad \alpha+\beta=1,
$$

in the same fashion as in the case of compactly supported perturbations. Here

$$
S_{p}(T)=\{(x, t): 0 \leqq t \leqq T, a \leqq x \leqq a+p\},
$$

$a$ is an arbitrary number and the constant is independent of $T$. The remaining terms on the right-hand side of (6.14) admit a bound which is quadratic in $\varepsilon$ and linear $T$, e.g.

$$
\beta_{1}=\left\{\lambda_{2}\left(w_{1}, w_{2}^{0}\right)-\lambda_{2}\left(w_{1}, w_{2}\right)\right\} \partial_{x} w_{1}
$$

satisfies

$$
\left|\beta_{1}\right|\left\{\mathscr{S}_{p}(T)\right\} \leqq \text { const } \int_{0}^{T} T V_{p}^{2} u(\cdot, t) d t \leqq \text { const } T \varepsilon^{2}
$$

by virtue of the basic total variation bound (5.8),

$$
T V_{p} u^{\varepsilon}(\cdot, t) \leqq \text { const } T V_{p} u^{\varepsilon}(\cdot, 0)=\text { const } \varepsilon .
$$

Taking into account the information in the refined estimate (6.15) does not lead to an improvement if one merely employs a quadratic bound for $\beta_{1}$ and optimizes with respect to a parameter $\tau$ in $[0, T]$ :

$$
\begin{aligned}
\left|\beta_{1}\right|\left\{\mathscr{S}_{p}(T)\right\} & \leqq \text { const } \int_{0}^{\tau} T V^{2} u(t) d t+\text { const } \int_{\tau}^{T} T V^{2} u(t) d t \\
& \leqq \text { const } \int_{0}^{\tau} \varepsilon^{2} T V^{2} u_{0} d t+\int_{\tau}^{T} \operatorname{const} \frac{\varepsilon^{2 \alpha}\left(T V u_{0}\right)^{2 \alpha}}{t^{2 \beta}} d t \\
& =\operatorname{const}\left\{\varepsilon^{2} \tau+\varepsilon^{2 \alpha} \frac{1}{\tau^{2 \beta-1}}\right\} \leqq \text { const } \varepsilon
\end{aligned}
$$

for the optimal choice of $\tau$.

\section{References}

1. Dafermos, C. M.: Characteristics in hyperbolic conservation laws. A Study of the structure and asymptotic behaviour of solutions. In: Nonlinear analysis and mechanics: Heriot-Watt Symposium, Vol. I, Knops, R. J., ed. Pitman Research Notes in Mathematics \#17

2. DiPerna, R. J.: Decay and asymptotic behavior of solutions to nonlinear hyperbolic systems of conservation laws, Indiana Univ. Math. J. 24, 1047-1071 (1975)

3. DiPerna, R. J.: Singularities of solutions of nonlinear hyperbolic systems of conservation laws. Arch. Rat. Mech. Anal. 60, 75-100 (1975)

4. DiPerna, R. J.: Uniqueness of solutions of hyperbolic conservation laws, Indiana Univ. Math. J. 28, 137-188 (1979)

5. Federer, H.: Geometric measure theory, Berlin, Heidelberg, New York: Springer, 1969 
6. Glimm, J.: Solutions in the large for nonlinear hyperbolic systems of equations. Commun. Pure Appl. Math. 18, 697-715 (1965)

7. Glimm, J., Lax, P. D.: Decay of solutions of systems of nonlinear hyperbolic conservation laws. Am. Math. Soc. Memoirs 101 (1970)

8. Hunter, J. K., Keller, J. B.: Weak shock diffraction. (in press, Wave Motion)

9. Hunter, J. K., Majda, A., Rosales, R.: Resonantly interacting weakly nonlinear hyperbolic waves, II: several space variables. (in preparation)

10. Hunter, J. K., Keller, J. B.: Weakly nonlinear high frequency waves. Commun. Pure Appl. Math. 36, 547-569 (1983)

11. Keyfitz, B.: Solutions with shocks, an example of an $L^{1}$-contractive semi-group. Commun. Pure Appl. Math, 24, 125-132 (1971)

12. Kruzkov, N.: First order quasilinear equations in several independent variables. Math. USSR Sb. 10, 127-243 (1970)

13. Landau, L. D.: On shock waves at large distances from their place of origin. J. Phys. USSR 9, 495-500 (1945)

14. Lax, P. D.: Hyperbolic systems of conservation laws and the mathematical theory of shock waves. CMBS Monograph No. 11, SIAM, 1973

15. Lax, P. D.: Shock waves and entropy. In: Contributions to nonlinear functional analysis, Zarantonello, E. ed. New York: Academic Press, 1971

16. Lax, P. D.: Accuracy and resolution in the computation of solutions of linear and nonlinear equations. In: Recent advances in numerical analysis, pp. 107-118. DeBoor, C., Golub, G. ed. New York: Academic Press, 1978

17. Lighthill, M. J.: A method for rendering approximate solutions to physical problems uniformly valid. Phil. Mag. 40, 1179-1201 (1949)

18. Liu, T.-P.: Admissible solutions to systems of conservation laws. Am. Math. Soc. Memoirs 1982

19. Liu, T.-P.: Decay to $N$-waves of solutions of general systems of nonlinear hyperbolic conservation laws. Commun. Pure Appl. Math. 30, 585-610 (1977)

20. Majda, A., Rosales, R.: Resonantly interaction weakly nonlinear hyperbolic waves, I: A single space variable, (in press Studies Appl. Math. 1984)

21. Majda, A.: Compressible fluid flow and systems of conservation laws in several space variables. Applied Mathematical Science Series. Berlin, Heidelberg, New York: Springer, 1984

22. Majda, A., Rosales, R.: A theory for spontaneous Mach stem formation in reacting shock fronts, $\mathrm{I}$ : the basic perturbation analysis. SIAM J. Appl. Math. 43, 1310-1334 (1983)

23. Majda, A., Rosales, R.: Weakly nonlinear detonation waves. SIAM J. Appl. Math. 43, 1086-1118 (1983)

24. Nayfeh, A. H.: A comparison of perturbation methods for nonlinear hyperbolic waves. In: Singular perturbations and asymptotics, pp. 223-276. Meyer, R., Parter, S. eds. New York: Academic Press, 1980

25. Vol'pert, A. I.: The spaces BV and quasilinear equations. Math. USSR Sb. 2, 257-267 (1967)

26. Whitham, G. R.: The flow pattern of a supersonic projectile. Commun. Pure Appl. Math. 5, 301-348 (1952)

Communicated by A. Jaffe

Received June 18, 1984 
